\title{
Bayesian nonparametric vector autoregressive models
}

\author{
Maria Kalli ${ }^{1}$, Jim E. Griffin ${ }^{*, 1}$ \\ School of Mathematics, Statistics \& Actuarial Science, University of Kent, Canterbury, UK
}

\section{A R T I C L E I N F O}

\section{Article history:}

Received 13 September 2015

Received in revised form 4 August 2017

Accepted 16 November 2017

Available online 12 January 2018

\section{JEL classification:}

C11

C15

C52

C53

C58

\section{Keywords:}

Vector autoregressive models

Dirichlet process prior

Infinite mixtures

Markov chain Monte Carlo

\begin{abstract}
A B S T R A C T
Vector autoregressive (VAR) models are the main work-horse models for macroeconomic forecasting, and provide a framework for the analysis of complex dynamics that are present between macroeconomic variables. Whether a classical or a Bayesian approach is adopted, most VAR models are linear with Gaussian innovations. This can limit the model's ability to explain the relationships in macroeconomic series. We propose a nonparametric VAR model that allows for nonlinearity in the conditional mean, heteroscedasticity in the conditional variance, and non-Gaussian innovations. Our approach differs from that of previous studies by modelling the stationary and transition densities using Bayesian nonparametric methods. Our Bayesian nonparametric VAR (BayesNP-VAR) model is applied to US and UK macroeconomic time series, and compared to other Bayesian VAR models. We show that BayesNP-VAR is a flexible model that is able to account for nonlinear relationships as well as heteroscedasticity in the data. In terms of short-run out-of-sample forecasts, we show that BayesNP-VAR predictively outperforms competing models.
\end{abstract}

(C) 2018 The Authors. Published by Elsevier B.V. This is an open access article under the CC BY license (http://creativecommons.org/licenses/by/4.0/).

\section{Introduction}

Introduced by Sims (1980), vector autoregressive (VAR) models provide a systematic way of capturing the dynamics and interactions of multiple time-series. In its basic form, the $L$-lag VAR model represents a $p$-dimensional vector of variables measured at time $t$, $y_{t}=\left(y_{t, 1}, \ldots, y_{t, p}\right)^{\prime}$, as a linear combination of past realisations,

$y_{t}=\mu+B_{1} y_{t-1}+\cdots+B_{L} y_{t-L}+e_{t}$

where $\left\{B_{l}\right\}_{l=1}^{L}$ are $(p \times p)$-dimensional matrices of unknown coefficients, and $e_{t}=\left(e_{1, t}, \ldots, e_{p, t}\right)^{\prime}$ is a $(p \times 1)$-dimensional innovation vector with distribution $\mathrm{N}(0, \Sigma)$. VAR models have emerged as a benchmark for the analysis of dynamic macroeconomic problems. The linear representation of the variables' joint dynamic behaviour facilitates the study of the effects of shocks (such as monetary and fiscal policy shocks) through computation of response functions, and forecast error variance decompositions (see Lucas, 1980; Pagan, 1997; Stock and Watson, 1999; Diebold and Rudebusch, 2001).

Despite their popularity, there have been criticisms of the use of VAR models in macroeconomic analysis. When $p$ is large there is

\footnotetext{
* Corresponding author.

E-mail addresses: M.Kalli-672@kent.ac.uk(M. Kalli),J.E.Griffin-28@kent.ac.uk (J.E. Griffin).

1 Acknowledgements: The authors would like to thank the Co-Editor and the two Referees for their helpful comments.
}

the risk of overfitting the data which leads to imprecise inference and erratic model forecasts. In addition, the linearity, stationarity, Gaussian innovations and constant conditional mean and variance of these models can be considered unrealistic. For example, empirical evidence suggests that macroeconomic variables may have nonlinear relationships (see Granger and Terasvirta, 1994), the nature of shocks may not be Gaussian (Weise, 1999), and the effects of these shocks may not be linear (see Ravn and Sola (2004) and Matthes and Barnichon (2015) for monetary policy studies, and Sörensen et al. (2001), Auerbach and Gorodnichenko (2013), Baum and Koester (2011), and Gambacorta et al. (2014) for fiscal policy studies).

In the last two decades these criticisms have been addressed by: using adaptations of the parametric model given in Eq. (1) such as regime switching and threshold crossing behaviour, introducing time varying coefficient models with or without stochastic volatility, and, more recently, using nonparametric methods and considering non-Gaussian innovations. Both regime switching and threshold models are motivated by empirical evidence that many macroeconomic time series behave differently during different time periods (for example, in economic downturns and in expansions) which are often called regimes. Both models assume that there are a small number of regimes which can be accurately modelled by different VARs. The mechanism for the change between regimes is the key difference between the two approaches. Hamilton (1989) popularised Markov-switching regression where the change in regimes is driven by latent (unobservable) stochastic 
variables, usually with a Markov structure. Literature on these models has subsequently grown, see e.g. Hansen (1992), Chib (1996), Chauvet (1998), Kim and Nelson (1999), Kim et al. (2005), and Sims and Zha (2006). Beaudry and Koop (1993), Teräsvirta (1994), Potter (1995), and Pesaran and Potter (1997) popularised vector threshold autoregressive (VTAR) and vector smooth transition autoregressive (VSTAR) models. Unlike Markov-switching regressions, regime changes in a VTAR model occur if some function (often, a linear function) of the observable macroeconomic variables crosses a threshold. Whereas VSTAR uses a weighted average of VAR models where the weighting depends on a continuous, non-linear function of the previous lags. For a comprehensive survey see Hubrich and Teräsvirta (2013). In contrast, time varying vector autoregressions are a class of models in which the system's conditional mean and/or variance are allowed to vary over time. This is achieved by modelling the VAR parameters (coefficients and innovation covariance matrix) with a linear time series model, often a random walk or an $\mathrm{AR}(1)$ process. Notable work in this area is presented in Stock and Watson (1996, 2001, 2002), Cogley and Sargent (2001, 2005a), and Primiceri (2005). See Koop and Korobilis (2010) for a recent review of these methods. An alternative approach to modelling the joint dynamic behaviour are nonparametric methods. Härdle et al. (1998) proposed a vector conditional heteroskedastic autoregressive nonlinear model where both the conditional mean and variance are unknown functions of past observations. Hamilton (2001) developed a flexible parametric regression model where the conditional mean has a linear parametric component and a potential nonlinear component represented by an isotropic Gaussian random field. Dahl and GonzalezRivera (2003a, b) extended his model to non-Gaussian random fields, while Jeliazkov (2013) models the conditional mean using a Bayesian hierarchical representation of generalised additive models, where a "smoothness prior" is given to the nonparametric function of the vector of past realisations. The use of non-Gaussian innovations is linked to structural VAR models where the computation of impulse response functions requires identification of the structural errors. Hyvärinen et al. (2010), Moneta et al. (2013), and Lanne et al. (2017) use independent component analysis where they assume mutual independence across the non-Gaussian innovation processes and represent the residuals (obtained when estimating the VAR model) as linear mixtures of these. Lanne and Lütkepohl (2010) model the innovations using a mixture of two Gaussian distributions, whereas Jeliazkov (2013) uses the Student t-distribution.

Geweke and Keane (2007) state that answering interesting questions in economics, from macroeconomic policy to the evaluation of economic welfare, often requires the entire conditional distribution $p(y \mid x)$. In this paper, we introduce a novel stationary model for multivariate time series where the stationary and transition densities are directly modelled using Bayesian nonparametric methods, which place a prior on an infinite dimensional parameter space and adapt their complexity to the data (see Hjort et al., 2010 for a book length review of Bayesian nonparametric methods). The Bayesian nonparametric approach to density estimation requires a prior on distributions with random smooth densities. We use a Dirichlet process mixture (DPM), the most popular of these priors, which is an infinite mixture model, with the Dirichlet process as the mixing measure. There are several advantages to using Bayesian nonparametric methods. Unlike classical nonparametric methods, there is no need to tune any smoothing parameters. Uncertainty in the unknown density can be expressed through the posterior. The out-of-sample predictive performance of models where the conditional density is estimated using the Bayesian nonparametric approach is superior to other competitive models, see Norets and Pati (2017). Adopting the DPM prior, allows us to construct a mixture of VARs which can be viewed as a multivariate mixture-of-experts. Introduced by Jacobs et al. (1991) and Jordan and Jacobs (1994), mixture-of-experts models focus on estimating the conditional predictive density $p(y \mid x)$ for all $x$ where $y$ is univariate (discrete or continuous) and $x$ a high dimensional set of covariates. They are extensions of mixture regression models that allow for covariates in the mixture weights. Geweke and Keane (2007) and Villani et al. (2009) provide extensive analyses when the mixture components are Gaussian, whereas Villani et al. (2012) allow for distributions outside the exponential family to represent the mixture components. In our Bayesian nonparametric mixture of VARs, the mixing weights of the transition density depend on the previous lags allowing different component transition densities to be favoured at different times (for example, in expansionary and contractionary periods) based on lagged observed values. Intuitively, we can view each mixture component ("expert") as a regime with changes of regime determined by the lagged values (through the mixing weights). Our Bayesian nonparametric VAR model allows for nonlinearity in the conditional mean, heteroskedasticity in the conditional variance, and nonGaussian innovations. We tackle over-parameterisation and the danger of overfitting in two ways, via a prior on the number of mixture components and by modelling the dependence within each component with a prior favouring a simple correlation structure. We find that our approach produces better forecasts (particularly at longer time horizons) when compared to the widely used time varying parameter models with stochastic volatility (TVP-SV-VAR).

The paper is organised as follows: Section 2 introduces the Bayesian non-parametric VAR (BayesNP-VAR) model, describes its construction and considers some of its properties. Section 3 provides an overview of the required Markov chain Monte Carlo (MCMC) method for fitting this model (the full steps of the MCMC sampler are described in Appendix A). Section 4 illustrates the ability of the BayesNP-VAR model to identify regimes and changes in regimes using simulated data, provides an empirical illustration using US macroeconomic time series, and compares the outof-sample predictive performance of the BayesNP-VAR to the parametric BVAR and TVP-SV-VAR using both US and UK macroeconomic series. Section 5 summarises our findings and conclusions.

\section{The Bayesian non-parametric vector autoregressive (BayesNP-VAR) model}

We construct a multivariate time series model in which the stationary and transition densities are infinite mixtures. AntonianoVillalobos and Walker (2016) define such a model for a univariate stationary time series. Their prior has full support for the transition density and stationary density (i.e. any transition density and stationary density can be represented arbitrarily well by the prior). We extend their work to multivariate stationary time series and we call our model Bayesian nonparametric VAR (BayesNP-VAR).

The transition densities of the BayesNP-VAR model are derived from a joint distribution for $y_{t}$ and its $L$ lags $y_{t}^{L}$ which is expressed as an infinite mixture. This ensures that the stationary distribution is known and also has the form of an infinite mixture. Specifically, the joint density of $y_{t}$ and its $L$ lags $y_{t}^{L}$ is

$p\left(y_{t}, y_{t}^{L}\right)=\sum_{j=1}^{\infty} w_{j} k\left(y_{t}, y_{t}^{L} \mid \theta_{j}\right)$

where $k\left(y_{t}, y_{t}^{L} \mid \theta_{j}\right)$ is a $(L+1) p$-dimensional probability density function which does not depend on $t$ and $\theta_{j}$ are the locations of the mixture components with $\theta_{j} \stackrel{\text { iid }}{\sim} H$. We assume that $k\left(y_{t-i}, \ldots, y_{t-i-\kappa} \mid \theta_{j}\right)$ for $i=0, \ldots, L-\kappa$ and $\kappa=0, \ldots, L-$ 1 depends on $\kappa$ only (which can be achieved by assuming that $k\left(y_{t}, y_{t}^{L} \mid \theta_{j}\right)$ is the joint distribution of a stationary process) to ensure 
that the overall process is stationary. The mixture weights $w_{j}$ are defined as $w_{1}=v_{1}, w_{j}=v_{j} \prod_{m<j}\left(1-v_{m}\right)$, and $v_{j} \stackrel{i i d}{\sim} \operatorname{Be}(1, M)$. Assuming that the locations $\theta_{j}$ are independent of the weights, $w_{j}$, the model in Eq. (2) defines a Dirichlet process mixture (Sethuraman, 1994). The distribution of the locations, $H$, is often referred to as the "base measure", the choice of which determines the likely location of the components. The parameter $M$ controls the relative values of the weights. The expectation of the $j$ th weight is $\mathrm{E}\left[w_{j}\right]=\frac{M^{j-1}}{(M+1)^{j}}$ and so, as $M$ increases, the average size of the $j$ th weight becomes smaller and the number of components with nonnegligible weights becomes larger. Choosing a prior for $M$ is key to controlling the number of components and avoiding overfitting, we discuss this choice later in this section.

The joint density in Eq. (2) leads to a transition density that is also an infinite mixture with the following form:

$$
\begin{aligned}
p\left(y_{t} \mid y_{t}^{L}\right)=\frac{p\left(y_{t}, y_{t}^{L}\right)}{p\left(y_{t}^{L}\right)} & =\frac{\sum_{j=1}^{\infty} w_{j} k\left(y_{t}, y_{t}^{L} \mid \theta_{j}\right)}{\sum_{j=1}^{\infty} w_{j} k\left(y_{t}^{L} \mid \theta_{j}\right)} \\
& =\sum_{j=1}^{\infty} \omega_{j}\left(y_{t}^{L}\right) k\left(y_{t} \mid y_{t}^{L}, \theta_{j}\right)
\end{aligned}
$$

where $k\left(y_{t} \mid y_{t}^{L}, \theta_{j}\right)$ is the transition density of the $j$ th component and $\omega_{j}\left(y_{t}^{L}\right)=\frac{w_{j} k\left(y_{t}^{L} \mid \theta_{j}\right)}{\sum_{k=1}^{\infty} w_{k} k\left(y_{t}^{L} \mid \theta_{k}\right)}$ is the weight of the $j$ th component which depends on previous lags, the key feature of our model. We can therefore refer to the transition density as a multivariate mixture of experts. Mixtures of experts are extensions of smooth regression models and popular within the machine learning community. They are used in regression to estimate the conditional density $p(y \mid x)$ of a univariate $y$ for all values of a (often, high-dimensional) covariate $x$, using mixtures where the component weights depend on a $x$, see Jacobs et al. (1991), Jordan and Jacobs (1994), Geweke and Keane (2007) and Villani et al. (2012). The weights of the transition density in Eq. (3) depend on the observed lagged values. This feature allows different component transition densities to be favoured in different periods. For example, expansionary and recessionary periods could have different transition densities. In our multivariate time series model, each component ("expert") can be viewed as a regime with changes of regime determined by the observed lagged values.

To complete the BayesNP-VAR we need to choose $k\left(y_{t}, y_{t}^{L} \mid \theta_{j}\right)$. Firstly, we find it easier to write $y_{t}=\mu+S \epsilon_{t}$ where $\mu$ is a $p$ dimensional location vector and $S=\operatorname{diag}\left(s_{1}, \ldots, s_{p}\right)$ is a scaling matrix. Then $k\left(y_{t}, y_{t}^{L} \mid \theta_{j}\right)=\left(\prod_{j=1}^{p} s_{j}\right)^{-(L+1)} k_{\epsilon}\left(S^{-1}\left(y_{t}-\mu\right),\left(S^{-1} \otimes\right.\right.$ $\left.\left.I_{L}\right)\left(y_{t}-1_{L} \mu\right)\right)$ where $k_{\epsilon}$ is the joint density of $\epsilon_{t}, \epsilon_{t}^{L}$. We propose a model which has a structure similar to a factor model and divides the variation of the data into a part which describes the dependence between variables and a part which is idiosyncratic to each variable. The assumed form is $k_{\epsilon}\left(\epsilon_{t}, \epsilon_{t}^{L}\right)=\mathrm{N}\left(0_{(L+1) p}, B+Q\right)$ where $0_{(L+1) p}$ is a $(L+1) p$-dimensional vector of 0 's, $B$ describes the dependence between variables over time and $Q$ describes the idiosyncratic variation over time. The form of $B$ is

$B=\sum_{z=1}^{q} P_{z} \otimes\left(\Lambda_{\cdot, z} \Lambda_{\cdot, z}^{T}\right)$

where

$P_{z}=\left(\begin{array}{cccc}1 & \rho_{z} & \ldots & \rho_{z}^{L} \\ \rho_{z} & 1 & \ldots & \rho_{z}^{L-1} \\ \vdots & \vdots & \ddots & \vdots \\ \rho_{z}^{L} & \rho_{z}^{L-1} & \ldots & 1\end{array}\right)$,

$-1<\rho_{z}<1$ for $z=1, \ldots, q$ and $\Lambda$ is a $(p \times q)$-dimensional matrix of loadings. The matrix $Q$ is
$Q=\left(\begin{array}{ccccc}\Sigma_{0} & \Sigma_{1} & \Sigma_{2} & \ldots & \Sigma_{L} \\ \Sigma_{1} & \Sigma_{0} & \Sigma_{1} & \ldots & \Sigma_{L-1} \\ \Sigma_{2} & \Sigma_{1} & \Sigma_{0} & \ldots & \Sigma_{L-2} \\ \vdots & \vdots & \vdots & \ddots & \vdots \\ \Sigma_{L} & \Sigma_{L-1} & \Sigma_{L-2} & \ldots & \Sigma_{0}\end{array}\right)$,

where $\Sigma_{l}=\operatorname{diag}\left(\frac{\rho_{1}^{\star}}{\xi_{1}}, \frac{\rho_{2}^{\star}}{\xi_{2}}, \ldots, \frac{\rho_{p}^{\star^{l}}}{\xi_{p}}\right)$ for $l=1, \ldots, L,-1<$ $\rho_{k}^{\star}<1$ and $\xi_{k}^{-1} \sim \operatorname{Ga}(v / 2, v / 2)$ for $k=1, \ldots, p$. This leads to a suitable choice of $k\left(y_{t}, y_{t}^{L}\right)$ for the BayesNP-VAR model to be stationary. The marginal distribution is $k_{\epsilon}\left(\epsilon_{t}\right)=\mathrm{N}\left(0, \Lambda \Lambda^{T}+\right.$ $\left.\operatorname{diag}\left(\xi_{1}^{-1}, \xi_{2}^{-1}, \ldots, \xi_{p}^{-1}\right)\right)$ and so the marginal distribution of $y_{t}$ is $\mathrm{N}\left(\mu, S \Lambda \Lambda^{T} S^{T}+\operatorname{diag}\left(s_{1}^{2} \xi_{1}^{-1}, s_{2}^{2} \xi_{2}^{-1}, \ldots, s_{p}^{2} \xi_{p}^{-1}\right)\right)$.

The component-specific parameters in the mixture model are $\theta=\left(\mu, S, \Lambda, \rho, \rho^{\star}, \phi, \delta\right)$ where $\rho=\left(\rho_{1}, \ldots, \rho_{q}\right), \rho^{\star}=\left(\rho_{1}^{\star}, \ldots\right.$, $\left.\rho_{p}^{\star}\right)$ and $\delta=\left(\delta_{1}, \ldots, \delta_{q}\right)$. We assume that $\mu, S, \Lambda, \rho, \rho^{\star}, \phi$, and $\delta$ are a priori independent with distribution $H$ which has density,

$$
\begin{aligned}
h\left(\mu, S, \Lambda, \rho, \rho^{\star}, \phi, \delta\right)= & h_{\mu}(\mu) \times h_{S}(S) \times h_{\Lambda}(\Lambda) \\
& \times h_{\rho^{\star}}\left(\rho^{\star}\right) \times h_{\rho}(\rho) \times h_{\phi}(\phi) \times h_{\delta}(\delta) .
\end{aligned}
$$

The parameters $\mu$ and $S$ are given informative prior densities to avoid the mixture model placing mass in areas which are not plausible. We choose $h_{\mu}(\mu)=\mathrm{N}\left(\mu \mid \mu_{0}, \Sigma_{0}\right)$. Both parameters can be chosen with prior information but we use the data dependent choices $\mu_{0}=\bar{y}_{t}$, and $\Sigma_{0}=1.5^{2} \widehat{\Sigma}$, where $\bar{y}_{t}$, and $\widehat{\Sigma}$ are sample mean and covariance matrix of $y_{t}$ in the empirical examples in this paper. This choice leads to a prior for $\mu$ which is slightly overdispersed relative to the distribution of the data, and so the components are located in regions within or close to the data. For the $(p \times p)$-dimensional scaling matrix, $S=\operatorname{diag}\left(s_{1}, s_{2}, \ldots, s_{p}\right)$, we choose the hierarchical prior

$s_{i}^{-1} \stackrel{\text { ind. }}{\sim} \mathrm{Ga}\left(a_{s}, \zeta_{i}\left(a_{s}-1\right)\right), \quad \zeta_{i} \stackrel{i i d}{\sim} \mathrm{Ga}(1,5)$.

The hyperparameter $\zeta_{i}$ is shared by all components and is an estimate of the overall scale of the $i$ th variable. This hierarchical structure allows different components to have similar scales, the $s_{i}$ 's, for each variable.

We use the multiplicative gamma process shrinkage prior of Bhattacharya and Dunson (2011) for $\Lambda$. This allows the complexity of $B$ to adapt to the data. Under this prior, $\Lambda_{i, z} \stackrel{i i d}{\sim} \mathrm{N}\left(0, \phi_{i, z}^{-1} \tau_{z}^{-1}\right)$, for $i=1, \ldots, p$ and $z=1, \ldots, q$ where $\phi_{i, z} \sim \mathrm{Ga}(v / 2, v / 2)$ and $\tau_{z}=\prod_{i=1}^{z} \delta_{i}$ with $\delta_{1} \sim \mathrm{Ga}(1,1)$ and $\delta_{z} \sim \mathrm{Ga}(3,1)$ for $z \geq 2$. The $\delta_{z}$ 's are independent, and the $\tau_{z}$ 's are viewed as the global shrinkage parameters of the columns, while $\phi_{i, z}$ 's are the local shrinkage parameters for the $z$ th column. As value of $\delta_{z}$ increases, so does the value of $\tau_{z}$ favouring smaller values of $\Lambda_{i, z}$.

The parameters $\rho$ and $\rho^{\star}$ control dependence across time in both $B$ and $Q$. We choose independent uniform priors on the range that implies stationarity and positive autocorrelation to give $h_{\rho}(\rho)=\prod_{z=1}^{q} \mathrm{U}\left(\rho_{z} \mid 0,1\right)$ and $h_{\rho^{\star}}\left(\rho^{\star}\right)=\prod_{i=1}^{p} \mathrm{U}\left(\rho_{i}^{\star} \mid 0,1\right)$ where $\mathrm{U}(\rho \mid 0,1)$ represents the density of a uniform distribution between 0 and 1 . For the prior of $M$, the parameter controlling the number of components, we choose the standard exponential distribution. We find that this choice strikes a balance between having too many and/or too few components.

\section{Inference in Bayesian NP-VAR}

The likelihood function can be derived from the transition in Eq. (3) to be defined as

$\prod_{t=L+1}^{T} p\left(y_{t} \mid y_{t}^{L}\right)$. 
Bayesian inference is complicated by the infinite sum in both the numerator and denominator which precludes the direct use of Markov chain Monte Carlo methods. Antoniano-Villalobos and Walker (2016) describe a Gibbs sampler for their univariate model but truncate the centring distribution for the stationary variance of each component away from zero. To avoid this truncation, we use an adaptive truncation method introduced by Griffin (2016) which adaptively truncates the infinite sum in the numerator and denominator and tends to avoid large truncation errors in the posterior. We define a truncation of the infinite model in Eq. (2) with $K$ mixture components which leads to a truncated transition density which has the form

$p_{K}\left(y_{t} \mid y_{t}^{L}\right)=\frac{\sum_{j=1}^{K} w_{j} k\left(y_{t}, y_{t}^{L} \mid \theta_{j}\right)}{\sum_{j=1}^{K} w_{j} k\left(y_{t}^{L} \mid \theta_{j}\right)}$

where $w_{j}=V_{j} \prod_{m<j}\left(1-V_{m}\right)$ and $V_{j} \stackrel{i i d}{\sim} \mathrm{Be}(1, M)$ and $\theta_{j} \stackrel{i i d}{\sim} H$. This finite mixture model defines a sequence of posteriors of the form

$\pi_{K}\left(\theta_{1: K}, \eta_{1: K} \mid y\right) \propto p_{K}\left(\theta_{1: K}, \eta_{1: K}\right) \prod_{t=L+1}^{T} p_{K}\left(y_{t} \mid y_{t}^{L}\right)$

where $\eta_{1: K}=\left(V_{1: K}, \zeta, M\right)$. The adaptive truncation method of Griffin (2016) uses an MCMC algorithm to sample from the posterior, $\pi_{K_{0}}\left(\theta_{1: K_{0}}, \eta_{1: K_{0}} \mid y\right)$, for a user-defined starting value, $K_{0}$, and then uses a sequential Monte Carlo method to sample from the sequence of posterior distributions $\pi_{K_{0}+1}\left(\theta_{1:\left(K_{0}+1\right)}, \eta_{1:\left(K_{0}+1\right)} \mid y\right)$, $\pi_{K_{0}+2}\left(\theta_{1:\left(K_{0}+2\right)}, \eta_{1:\left(K_{0}+2\right)} \mid y\right), \ldots, \pi_{D}\left(\theta_{1: D}, \eta_{1: D} \mid y\right)$ where $D$ is chosen to avoid large truncation errors. The adaptive truncation scheme follows the algorithm below.

1. Simulate a sample of size $N$ using the MCMC sampler from $\pi_{K_{0}}\left(\theta_{1: K_{0}}, \eta_{1: K_{0}} \mid y\right)$ which will be denoted $\left(\theta_{1: K_{0}}^{(1)}, \eta_{1: K_{0}}^{(1)}\right), \ldots$, $\left(\theta_{1: K_{0}}^{(N)}, \eta_{1: K_{0}}^{(N)}\right)$ and set $K=K_{0}+1$.

2. Simulate $\left(\theta_{K}^{(i)}, \phi_{K}^{(i)}, V_{K}^{(i)}\right)$ from their prior distribution for $i=$ $1, \ldots, N$.

3. Evaluate

$$
\psi_{i}=\frac{\pi_{K}\left(\theta_{1: K}^{(i)}, \eta_{1: K}^{(i)} \mid y\right)}{\pi_{K-1}\left(\theta_{1:(K-1)}^{(i)}, \eta_{1:(K-1)}^{(i)} \mid y\right)}, \quad i=1, \ldots, N
$$

4. Evaluate

$$
\mathrm{ESS}_{K}=\frac{\left(\sum_{i=1}^{N} \psi_{i}\right)^{2}}{\sum_{i=1}^{N} \psi_{i}^{2}}
$$

5. If $\mathrm{ESS}_{K}<c N$ (we use $c=0.5$ ) then generate $N$ values where $\left(\theta_{K}^{(i)}, \eta_{K}^{(i)}\right)$ is sampled with probability proportional to $\psi_{i}$. Set $\psi_{i}=1$ for $i=1, \ldots, N$ and run one iteration of the MCMC sampler updating $\left(\theta_{1: K}^{(i)}, \eta_{1: K}^{(i)}\right)$ from $\pi_{K}\left(\theta_{1: K}, \eta_{1: K} \mid y\right)$ for $i=1, \ldots, N$.

6. Let $\Delta_{K}=\left|\mathrm{ESS}_{K}-\mathrm{ESS}_{K-1}\right|$. If $\Delta_{K} \leq \varepsilon, \Delta_{K-1} \leq \varepsilon$ and $\Delta_{K-2} \leq$ $\varepsilon$ terminate (we choose $\varepsilon$ equal to $0.001 \mathrm{~N}$ ). Otherwise, set $K=K+1$, and return to step 2 .

Full details of the MCMC algorithm are provided in Appendix A. The MCMC algorithm uses two types of adaptive MetropolisHastings algorithm which are briefly reviewed here. The first is the adaptive random walk Metropolis-Hastings algorithm (Atchadé and Rosenthal, 2005) with a normal proposal whose variance is updated during the running of the chain. Suppose that $\sigma_{t}^{2}$ is the proposal variance used at iteration $t$, then the proposal variance at time $t+1$ is $\sigma_{t+1}^{2}=\sigma_{t}^{2}+t^{-0.6}\left(\alpha_{t}-0.234\right)$ where $\alpha_{t}$ is the acceptance probability in the Metropolis-Hastings algorithm at the tth iteration. Atchadé and Rosenthal (2005) show that this algorithm is ergodic. The second algorithm is Adaptive scaling within the Adaptive Metropolis-Hastings (ASWAM) algorithm (Andrieu and Moulines, 2006; Atchadé and Fort, 2010). This is suitable for updating multiple parameters jointly. Suppose we wish to sample a parameter, $\lambda$, then the proposed value $\lambda^{\prime}$ at the $t$ th iteration is

$\lambda^{\prime} \sim \mathrm{N}\left(\lambda, s_{t}^{2} \Sigma_{t}\right)$

where $s_{t}$ is a scalar and $\Sigma_{t}$ is the sample covariance matrix of the first $t-1$ sampled values of $\lambda$ sampled from the posterior. The scale $s_{t}$ is updated using the recursion $s_{t+1}=s_{t}+t^{-0.6}\left(\alpha_{t}-0.234\right)$ where, again, $\alpha_{t}$ is the acceptance probability of the MetropolisHastings algorithm at the $t$ th iteration.

\section{Illustrations}

In this section we apply the BayesNP-VAR model to both simulated and empirical data. Our aim is to demonstrate that our model identifies economic regimes where shocks are transmitted in different ways, that it clearly indicates changes between regimes, and provide evidence of the model's good out-of-sample predictive performance.

To provide a point estimate of our mixture model, we approximate the posterior mode by selecting the MCMC sample which has the highest posterior density value. We refer to this as the posterior modal sample. This allows us to illustrate the model's ability to correctly identify regimes by producing time plots showing how the weights in the mixture model in Eq. (3) change over time and to highlight the component/regime which is favoured in a particular time period (by finding the component with the highest posterior weight). Since the transition density within each component is a VAR model, we also plot impulse response functions (IRFs) to a unit shock in a chosen variable for each component to understand the different ways that shocks are transmitted under different components/regimes. The usual methodology for generating IRFs is used which involves a polynomial function of the estimated VAR parameters.

The posterior mode is useful for presenting the inference from our model but it ignores posterior uncertainty. To provide IRFs which include posterior uncertainty, we also produce the IRFs suggested by Koop et al. (1996) which we will refer to as Generalised IRFs (GIRFs). These are used to study the effect of a shock of size $v$ at time $t$ on the state of the system at time $t+n$, given that there are no other shocks to the system after time $t$. They are defined as follows:

$$
\begin{aligned}
& \mathrm{GI}_{y}\left(n, v, Y_{t-1}\right) \\
& =\mathrm{E}\left[Y_{t+n} \mid e_{t}=v, e_{t+1}=0, \ldots, e_{t+n}=0, Y_{t-1}\right] \\
& \quad-\mathrm{E}\left[Y_{t+n} \mid e_{t}=0, e_{t+1}=0, \ldots, e_{t+n}=0, Y_{t-1}\right]
\end{aligned}
$$

for $n=1,2,3, \ldots$, where $e_{t}, e_{t+1}, \ldots, e_{t+n}$ represent the arbitrary shocks at times $t, t+1, \ldots, t+n$ and the expectations are taken conditional on the parameter values. This allows us to look at posterior distributions of $\mathrm{GI}_{y}\left(n, v, Y_{t-1}\right)$ to assess uncertainty in their estimation.

For the out-of-sample predictive performance illustration we calculate the log-predictive score (LPS) for all the variables (using their joint predictive distribution) and for each variable separately (using their marginal predictive distribution). We also calculate the root mean squared error (RMSE) for each variable. The joint LPS is given by

$-\sum_{i=s}^{T-h} \log p\left(y_{i+h} \mid y_{1}, \ldots, y_{i}\right)$ 
Table 1

US data; Source: FRED

\begin{tabular}{llll}
\hline Name & Description & Growth rates & Levels \\
\hline UNRATE & Unemployment rate & None & None \\
PCEPI & Personal Consumption Expenditure Index: $2009=100$ & $1200 \ln \left(\frac{y_{t}}{y_{t-1}}\right)$ & $1200 \ln \left(y_{t}\right)$ \\
PAYEMS & Total non-farm payroll, thousands of persons & $1200 \ln \left(\frac{y_{t}}{y_{t-1}}\right)$ & $1200 \ln \left(y_{t}\right)$ \\
FEDFUNDS & Federal funds rate & None & None \\
INDPRO & Industrial production index: $2012=100$ & $1200 \ln \left(\frac{y_{t}}{y_{t-1}}\right)$ & $1200 \ln \left(y_{t}\right)$ \\
LTIR & Long term interest rate & None & None \\
\hline
\end{tabular}

Table 2

UK data; Source: OECD.

\begin{tabular}{llll}
\hline Name & Description & Growth rates & Levels \\
\hline UNRATE & Unemployment rate & None & None \\
CPI & Consumer price Index: $2010=100$ & $1200 \ln \left(\frac{y_{t}}{y_{t-1}}\right)$ & $1200 \ln \left(y_{t}\right)$ \\
STIR & Short term interest rate & None & None \\
INDPRO & Industrial production index: $2010=100$ & $1200 \ln \left(\frac{y_{t}}{y_{t-1}}\right)$ & $1200 \ln \left(y_{t}\right)$ \\
LTIR & Long term interest rate & None & None \\
\hline
\end{tabular}

where $T$ is the size of the time series, $s$ is the time from where the prediction starts, and $h$ is the predictive horizon. Similarly, the marginal LPS for the $j$ th variable is given by

$$
-\sum_{i=s}^{T-h} \log p\left(y_{i+h, j} \mid y_{1}, \ldots, y_{i}\right) \text {. }
$$

The RMSE of the jth variable is given by

$$
\sqrt{\frac{1}{T-h-s+1} \sum_{i=s}^{T-h}\left(y_{i+h, j}-\mathrm{E}\left(y_{i+h, j} \mid y_{1}, \ldots, y_{i}\right)\right)^{2}} .
$$

For both measures, smaller scores indicate better predictive performance. We looked at $h=1,2$, and 4 months. We compare the out-of-sample predictive performance of our BayesNP-VAR model with other Bayesian VAR specifications: the stationary BVAR model with the independent Normal-Wishart prior (with either one, two, three or four lags), and the non-stationary non-linear TVPVAR model with stochastic volatility (TVP-SV-VAR) of Primiceri (2005) (with one lag). The BayesNP-VAR was chosen to have one lag.

\subsection{Simulated data}

The following simulated example illustrates the ability of the BayesNP-VAR to correctly identify regimes and the timing of regime switches. We generated data from a threshold $\operatorname{VAR}(2)$ model with $p=3$ variables and 500 time points. The data had two regimes and followed the VAR in Regime 1 if $y_{t-1,1}>0$ and Regime 2 otherwise. The two regimes were

- Regime 1

$$
\begin{aligned}
y_{t}= & \left(\begin{array}{c}
1.8 \\
0.52 \\
0.29
\end{array}\right)+\left(\begin{array}{ccc}
0.5 & 0.15 & 0 \\
0.20 & 0.34 & 0 \\
0.03 & 0.05 & 0.24
\end{array}\right) y_{t-1} \\
& +\left(\begin{array}{ccc}
0.15 & 0.20 & 0.80 \\
0.14 & -0.18 & 0.30 \\
0.07 & -0.03 & 0.14
\end{array}\right) y_{t-2}+e_{t}
\end{aligned}
$$

with covariance matrix,

$$
\Sigma_{1}=\left(\begin{array}{lll}
0.28 & 0.03 & 0.07 \\
0.03 & 0.29 & 0.14 \\
0.07 & 0.14 & 0.36
\end{array}\right)
$$

- Regime 2

$$
\begin{aligned}
y_{t}= & \left(\begin{array}{c}
-1.8 \\
0.32 \\
0.12
\end{array}\right)+\left(\begin{array}{ccc}
0.6 & -0.05 & 0.2 \\
0.20 & 0.09 & 0 \\
0.05 & 0 & 0.42
\end{array}\right) y_{t-1} \\
& +\left(\begin{array}{ccc}
0.21 & -0.10 & 0.05 \\
0.07 & 0.32 & 0 \\
0.06 & -0.02 & 0.45
\end{array}\right) y_{t-2}+e_{t}
\end{aligned}
$$

with covariance matrix,

$$
\Sigma_{2}=\left(\begin{array}{ccc}
0.54 & 0.06 & 0.02 \\
0.06 & 0.46 & 0.24 \\
0.02 & 0.024 & 0.56
\end{array}\right)
$$

Fig. 1 displays plots of the three simulated series and some results for the posterior modal sample which identifies two components with non-negligible mixing weights. The top row of each plot shows the time series for each variable and periods for which a component has the highest weight. The second row of plots shows the weight for that regime (and is the same for all variables). The threshold is indicated by a dashed line and was set at $y_{t-1,1}=0$. We can clearly see that the estimated regimes change correctly as the value of $y_{t-1,1}$ changes. The second row displays the weight of the regime and we can see that the regimes are correctly identified with probability close to 1 .

We also simulated a VAR(2) model with $p=3$ variables and 500 time points with the specification given in Appendix B. We do not display the results here but our model is able to correctly identify that there is only one regime. Both simulated threshold VAR(2) and $\operatorname{VAR}(2)$ data are used in Section 4.3 as part of our out-of-sample forecasting exercise.

\subsection{Empirical examples}

\subsubsection{Data sets}

We constructed two macroeconomic data sets, one for the US and one for the UK, based on the series described and transformations carried out in Carriero et al. (2015). Both data sets include seasonally adjusted monthly time series, the sample period for the US is from 1st January 1959 to 1st August 2016, and for the UK from 1st January 1978 to 1st February 2015. The US series were collected from the Federal Reserve Bank of St Louis (FRED) and UK series from the Organisation for Economic Cooperation and Development (OECD). Details of the variables together with the transformations used are displayed in Table 1 for the US and Table 2 for the UK respectively. We include the series in both growth rates and levels. 


\section{Component/Regime 1}
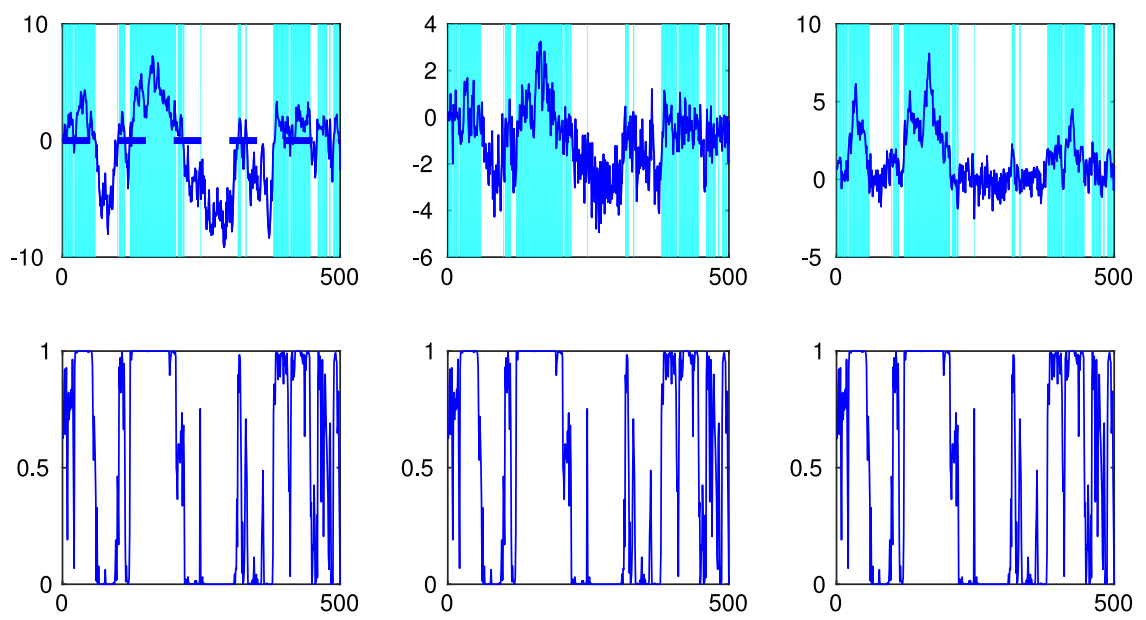

\section{Component 2/Regime 2}
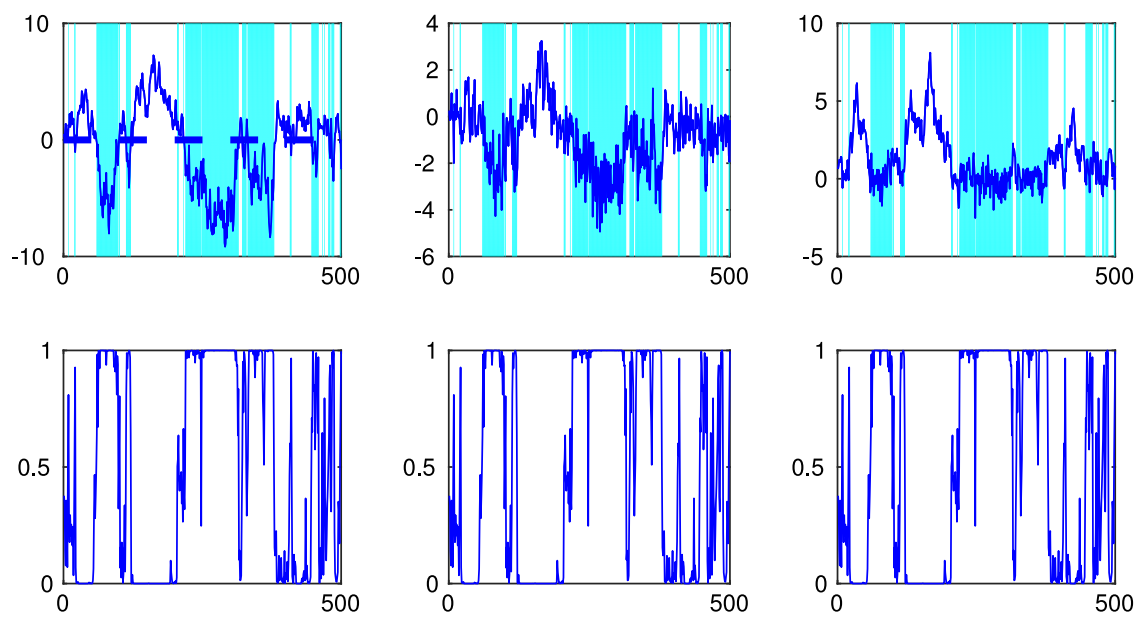

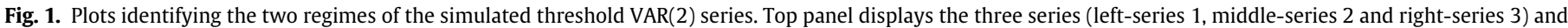
highlights (in cyan) the regime. The dashed line at zero in the series 1 plot is the threshold. Bottom panel displays the non-negligible weight of that regime.

For the illustrations in Sections 4.2 .2 and 4.2 .3 we used the US data, in growth rates. For the out-of-sample predictive exercise we have used both the US and UK data in growth rates and levels.

\subsubsection{Component/Regime identification}

Applying our BayesNP-VAR model to the US data in growth rates, we identify eight mixture components, and here we present the six which had the largest non-negligible weights. Figs. 2-4 display results for the six distinct components. The first two rows show the time series for each variable with the component/regime with the largest mixture weight and the weight for that component/regime is plotted over time (in the bottom row). The first regime, which we named, stable inflation (PCEPI-growth) and output (INDPROD) growth, covers periods of sustained growth and includes "The Great Moderation" of the mid-1980s to the mid2000 s, a period when volatility of business cycle fluctuations was low. The second component identifies periods after recent stock market crashes, caused by the burst of the 'Dot.com' bubble and the collapse of the US housing market respectively. These are periods of very low federal funds rates and long term interest rates with fairly stable growth and declining unemployment rate. These are in line with the National Bureau of Economic Research (NBER) peak to through periods of March 2001 to November 2001, and
November 2007 to June 2009 respectively. The "Golden Era" of US capitalism, the period up to the early 1970s, is captured by the third component, whereas the fourth component captures the period of mid 1981 to 1984 of the "Volcker disinflation". The last two components identify two of the worst recessions in the US where output growth was in decline and unemployment rapidly increasing. The "US housing crisis" of 2007 is captured by the fifth component, and the "Oil Shock" of the early 1970s is captured by the sixth component. The difference between the two is that the latter was characterised by high inflation.

\subsubsection{Impulse response functions}

Each component of our BayesNP-VAR model follows a VAR and so we can produce component/regime dependent IRFs as polynomial functions of the estimated VAR parameters. All IRFs look at 60 months ahead. Figs. 5 and 6 display these IRFs for each component using a different colour and pattern for each. The colour and pattern scheme is: blue dash inverted triangle is for the first component, red dash for the second, green dot dash for the third, yellow solid for the fourth, cyan circle dash for the fifth and pink plus dash for the sixth component. Recall that periods of stable inflation and output growth are in the first component, periods after the stock market crashes of 2001 and 2007 are in 
Component 1: Stable inflation and output growth

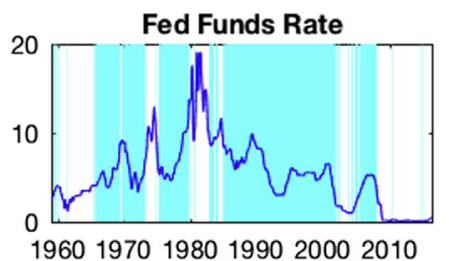

PAYEMS Growth
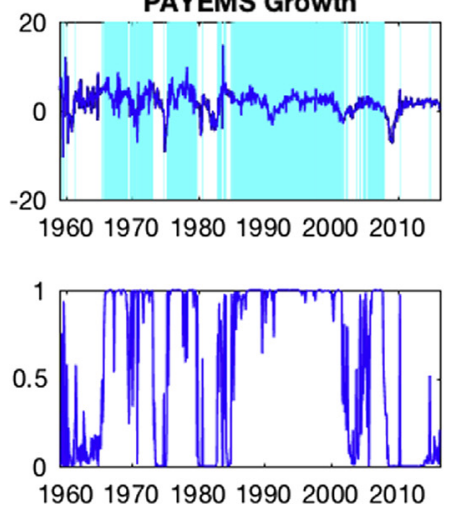

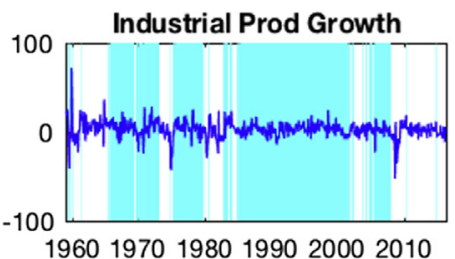

PCEPI Growth
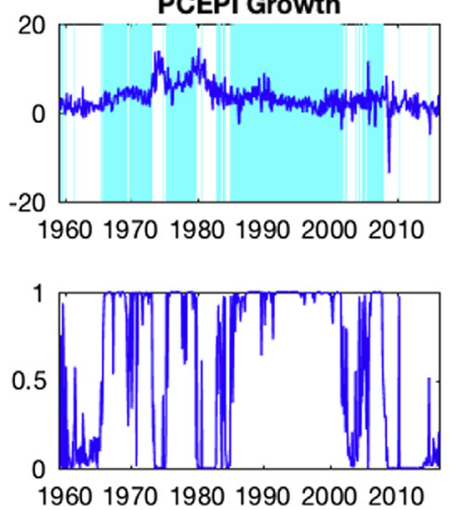

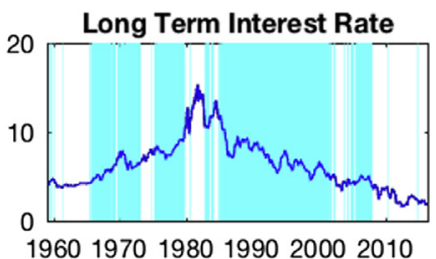

Unemployment Rate
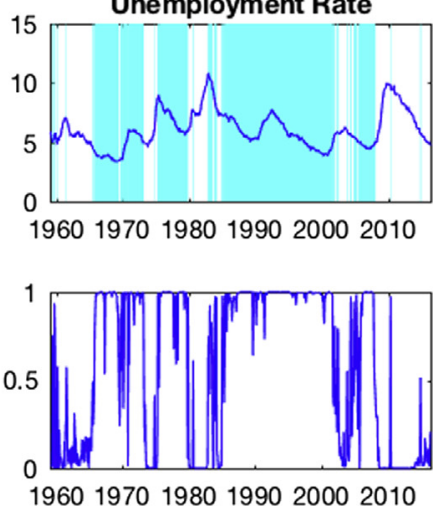

Component 2: After recent stock market crashes
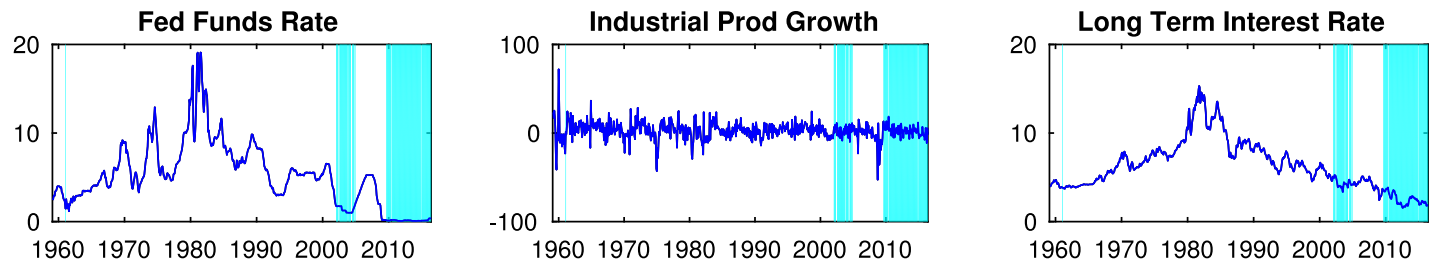

PAYEMS Growth
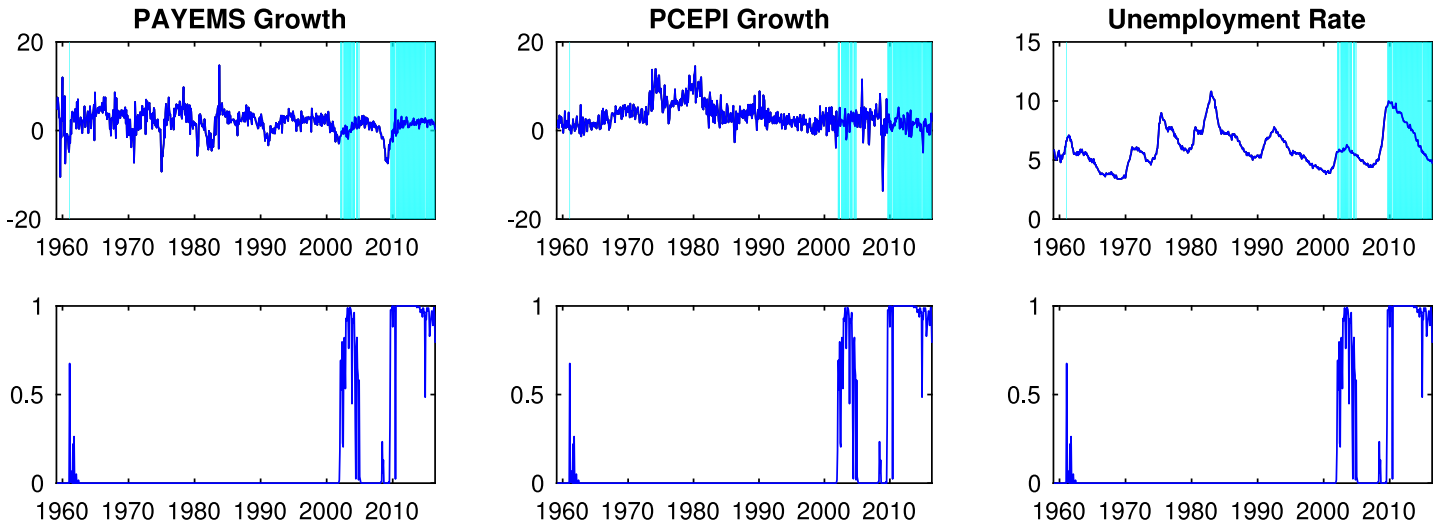

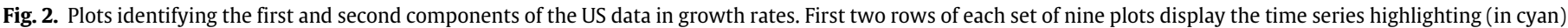
the component/regime, and the third row displays the non-negligible weight of the respective regime.

the second, the "Golden Era" periods in the third, the "Volcker disinflation" periods in the fourth, the periods of the US housing crisis in the fifth and the 1970s "Oil shock" is the sixth component. We centre our IRFs around the study of monetary policy. Fig. 5 displays the IRFs of inflation panel (a), output growth panel (b), and unemployment rate panel (c) to a $1 \%$ increase in the federal funds rate. It is clear that the IRFs are regime dependent with the transmission of the monetary policy shock differing between periods of expansion, stability and recession. Inflation eventually goes down and output growth declines in the two crisis periods of 1973 and 2007, whereas the monetary policy shock has small effect on inflation during the periods after the stock market crashes of 2001 and 2007, Volcker's chairmanship and the "Golden Era". During periods of relative stability inflation and output growth increase marginally before remaining at a constant level. The unemployment rate response steadily increases for the "Golden Era" and 2007 crisis, is relatively flat for the "Oil shock" and "Volcker disinflation" regimes, and declines for the component identifying the periods after the stock market crashes of 2001 and 2007, and the component identifying periods of relative economic stability. Fig. 6 displays the IRFs of the federal funds rate to a $1 \%$ increase in inflation panel (a) and to a $1 \%$ increase in unemployment rate panel (b). During the Volcker disinflation regime, the rate reacts quickly to both an inflationary and an unemployment shock, though the 
Component 3: Golden era
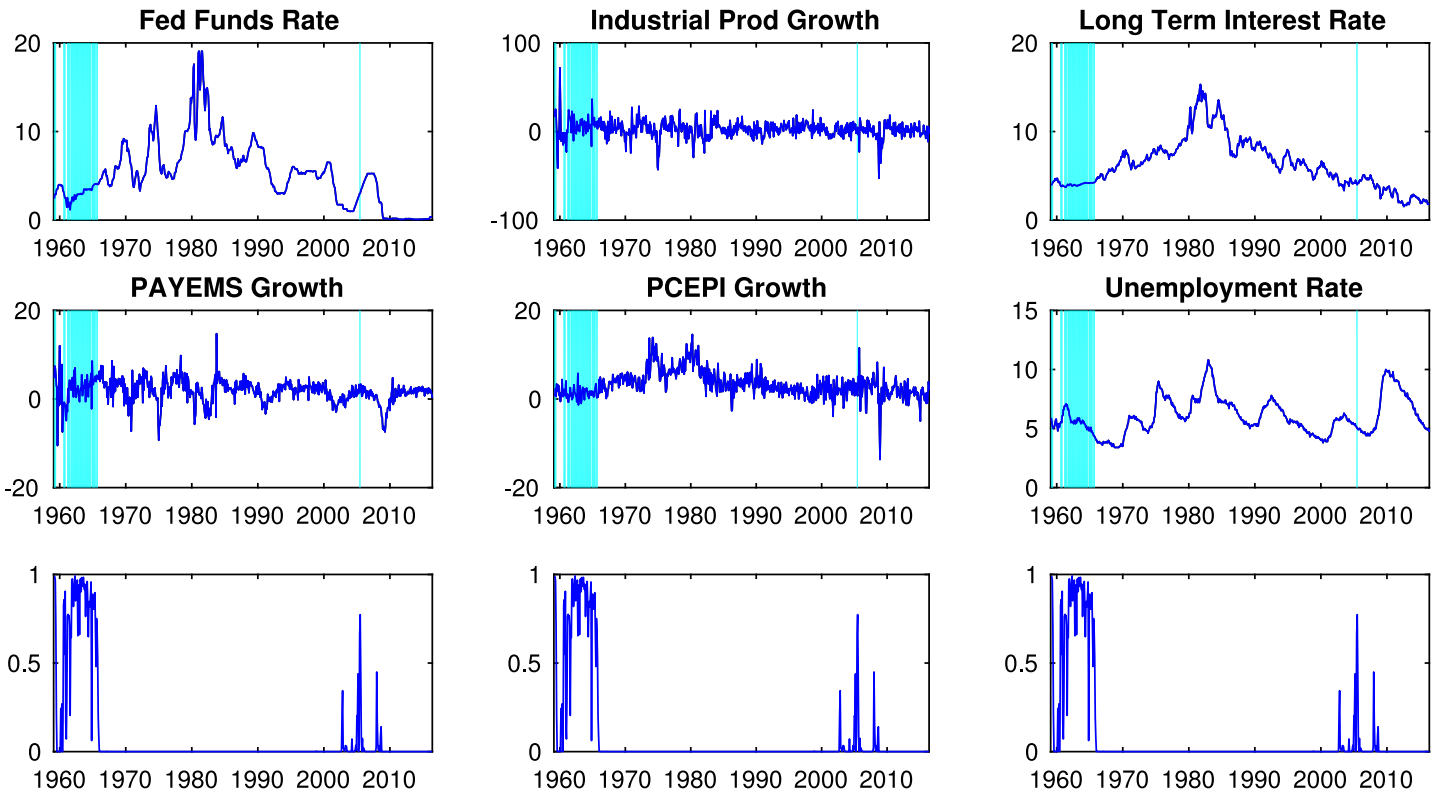

Component 4: Volcker disinflation
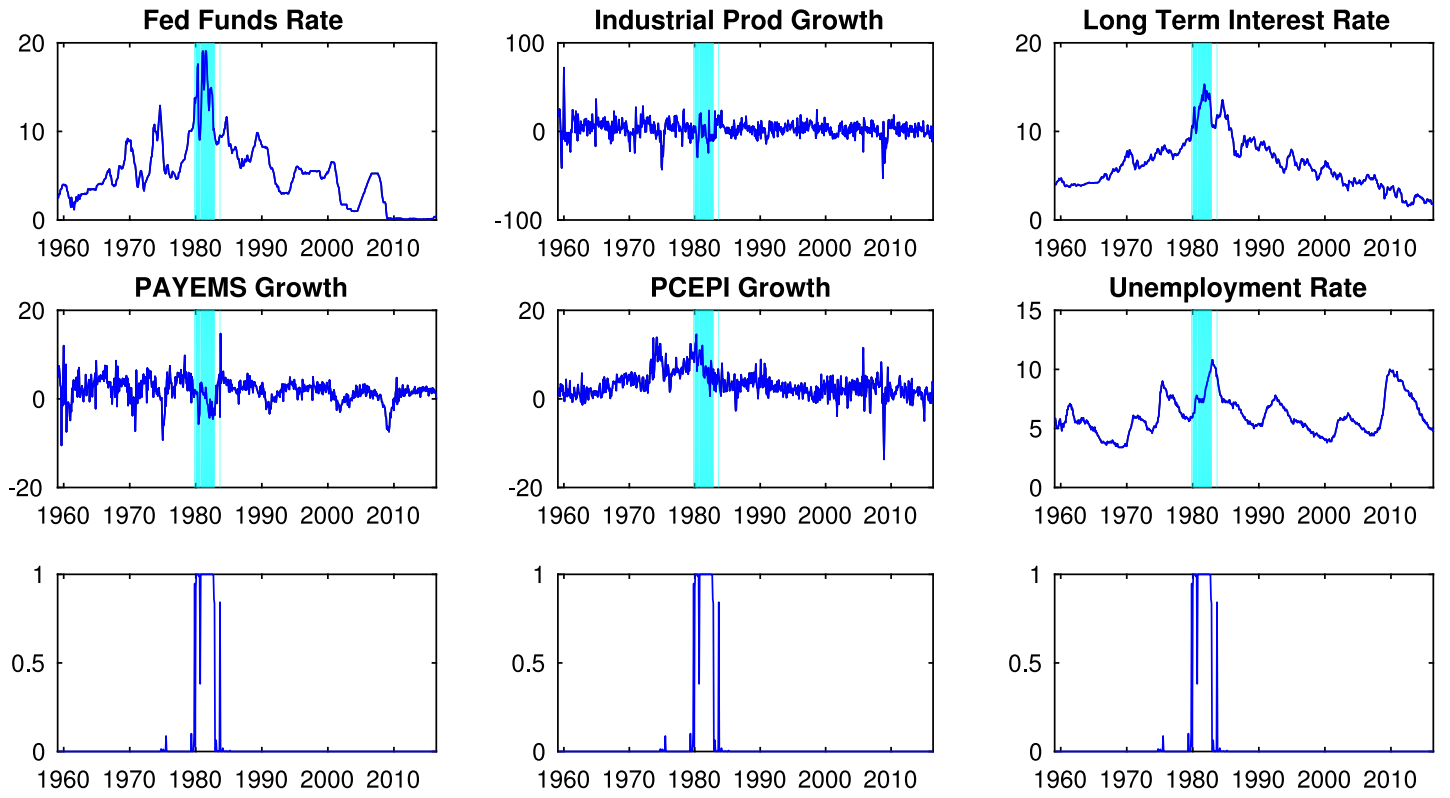

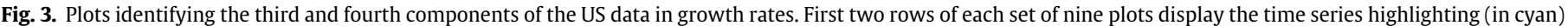
the component/regime, and the third row displays the non-negligible weight of the respective regime.

length of time before stabilising is longer for the latter (20 rather than 10 months). A similar pattern can be seen in the "Golden Era" regime, however the response to the unemployment shock is more prolonged with a sharp decline for the first 5 months. The rate response to the inflationary shock is not as extreme. When it comes to the other four regimes the inflationary shock has little impact, but the unemployment shock leads to a steady decline in the federal funds rate in the period of stable inflation and output growth, and to a marginal decline for the first 5 months before levelling off for the 2007 US housing crisis regime.

Figs. 7 and 8 display the GIRFs (see Eq. (5)) with their 95\% credible intervals for inflation and unemployment rate after a $1 \%$ increase in the federal funds rate at two different dates. The dates chosen for comparison are June 1981 and December 2007, the former because it is representative of Paul Volcker's chairmanship of the Fed and the latter because it is the beginning of the US housing crisis. Fig. 7 displays the response of inflation panel (a) and unemployment panel (b), over a 60 month period, to a $1 \%$ permanent increase in the Federal funds rate occurring in June 1981. There is no evidence of an effect. Fig. 8 draws the same graphs for a $1 \%$ increase in the Federal funds rate occurring in December 2007. There is clear evidence of an increase in inflation with the median response being a $0.5 \%$ increase. There is evidence of a negative effect on unemployment with the median effect close to zero, but the lower credible interval shows that a 1\% drop in unemployment is plausible. 
Component 5: The 2007 housing market crash
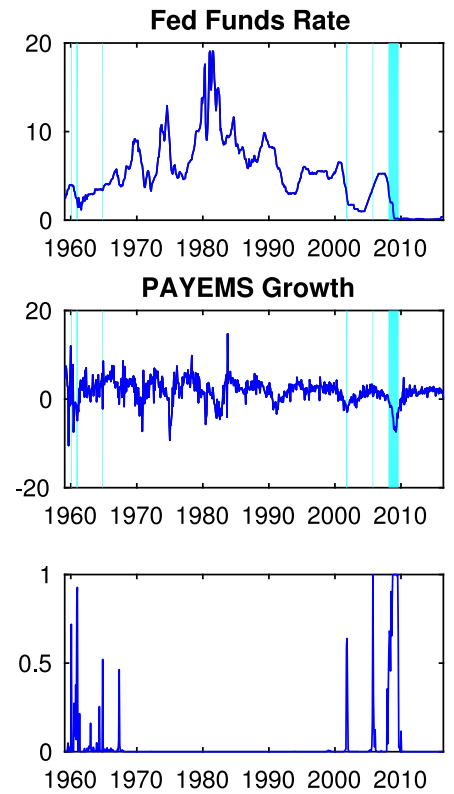
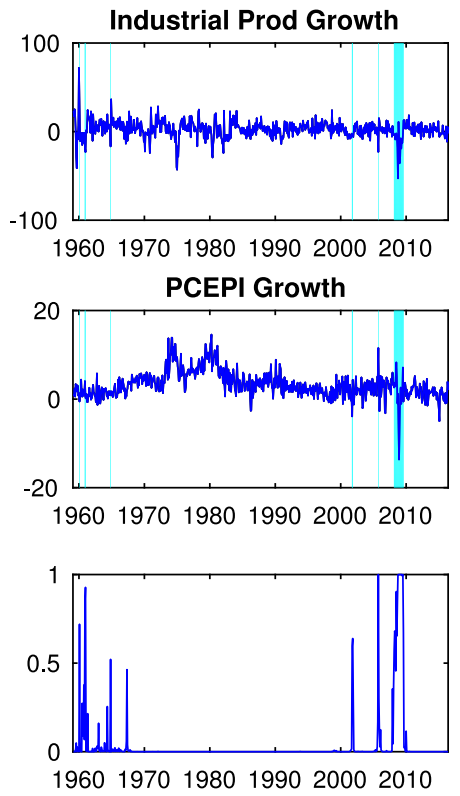
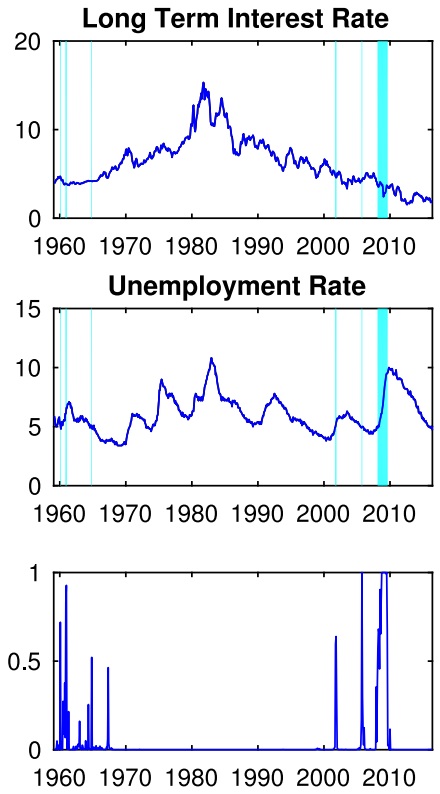

Component 6: The 1973 oil crisis
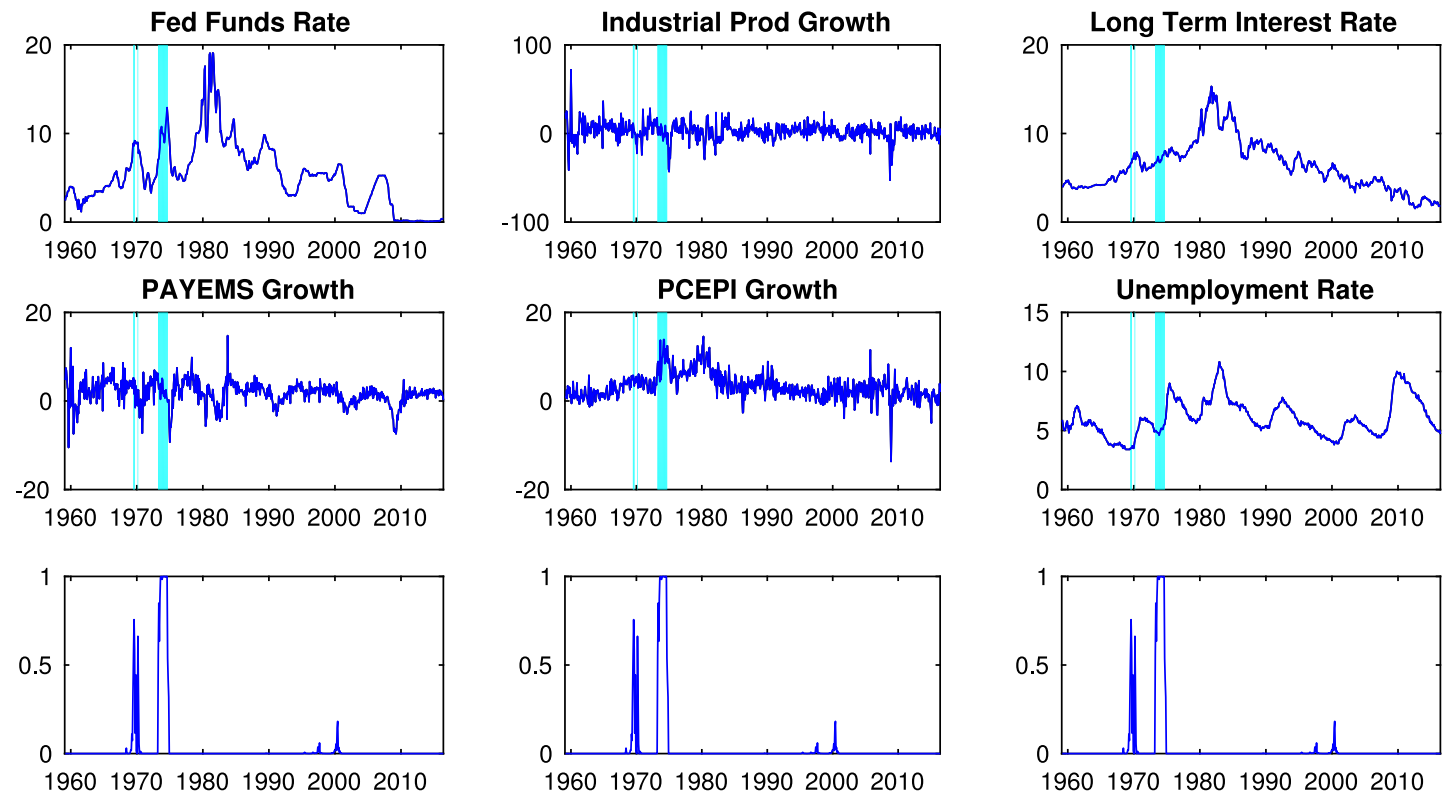

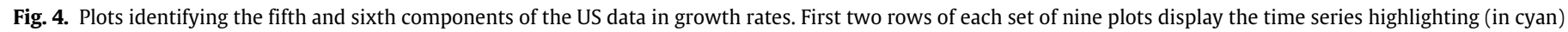
the component/regime, and the third row displays the non-negligible weight of the respective regime.

\subsection{Out-of-sample predictive performance}

We compare the out-of-sample predictive performance of the BayesNP-VAR model with other Bayesian VAR specifications: the stationary BVAR model with the independent Normal-Wishart prior (with one, two, three or four lags), and the non-stationary, non-linear TVP-VAR model with stochastic volatility (TVP-SV-VAR) of Primiceri (2005) (with one lag). Our comparison metrics are the log-predictive scores described in Eqs. (6) and (7) and the root mean squared error in Eq. (8). We used two simulated (from threshold $\operatorname{VAR}(2)$ and $\operatorname{VAR}(2)$ models) and four real data sets (US and UK data in growth rates and levels). We consider three predictive horizons: 1 month, 2 months and 4 months, and look at 48 months out-of-sample. This means that for the US data the prediction starts on 1st September 2012, and for the UK data on 1st March 2011.

Tables 3 and 4 display the log-predictive scores and the RMSEs for the simulated $\operatorname{VAR}(2)$ data and simulated threshold $\operatorname{VAR}(2)$ data respectively. The BayesNP-VAR(1) outperforms the TVP-SV$\operatorname{VAR}(1)$ for all predictive horizons both for all variables jointly and each variable marginally, in both the threshold $\operatorname{VAR}(2)$ and $\operatorname{VAR}(2)$ simulated data. In the VAR(2) data, the overall log-predictive scores and the RMSEs for the BVAR(1) and the BayesNP-VAR(1) are comparable at all horizons. In the threshold VAR(2) data, the BayesNP$\operatorname{VAR}(1)$ has the lowest overall log-predictive scores. It also 
(a)

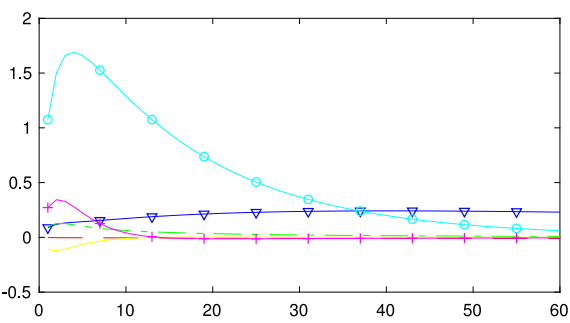

(b)

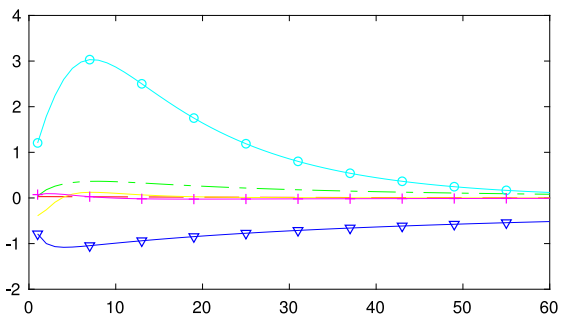

(c)

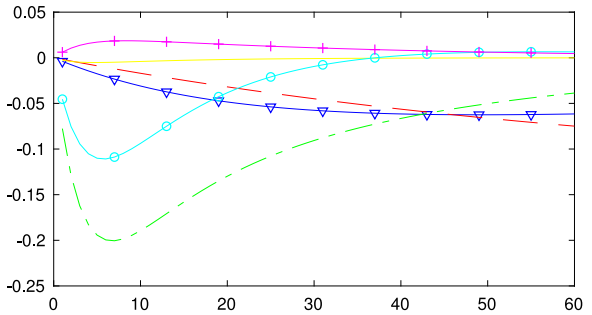

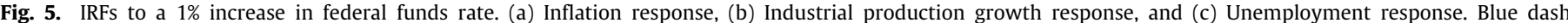

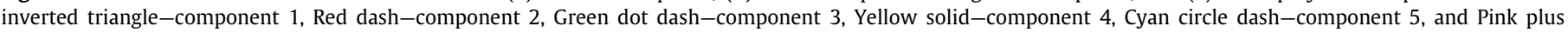
dash-component 6 .

(a)

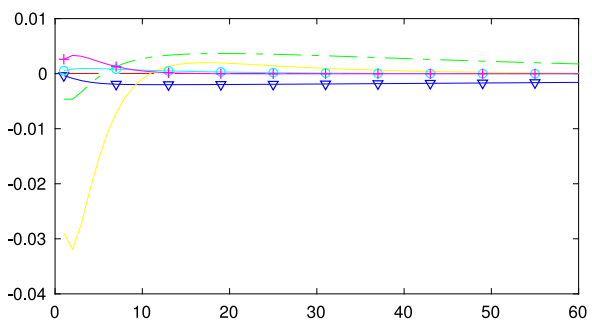

(b)

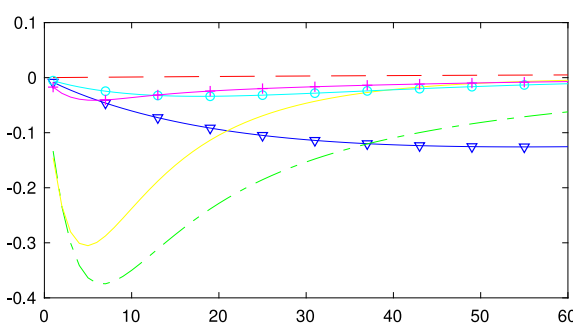

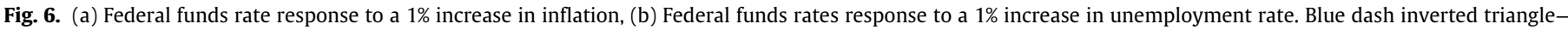
component 1, Red dash-component 2, Green dot dash-component 3, Yellow solid-component 4, Cyan circle dash-component 5, and Pink plus dash-component 6.

(a)

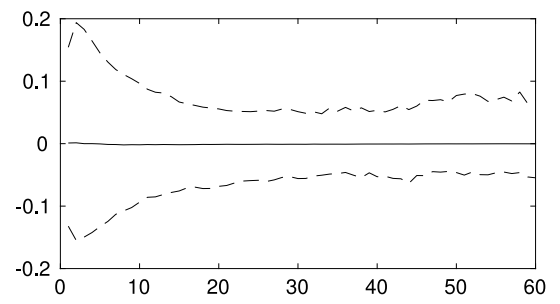

(b)

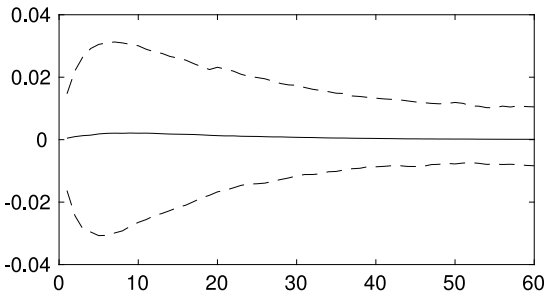

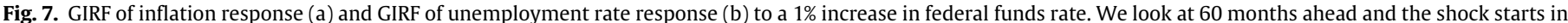
June 1981 . Solid line is the 50th percentile and dashed line the $95 \%$ credible interval.

(a)

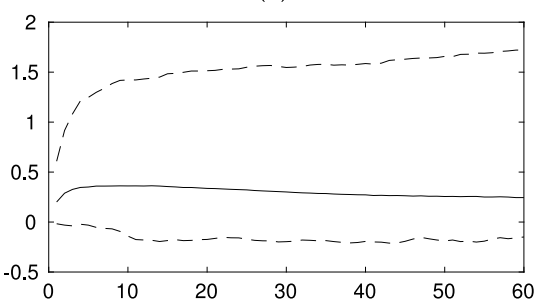

(b)

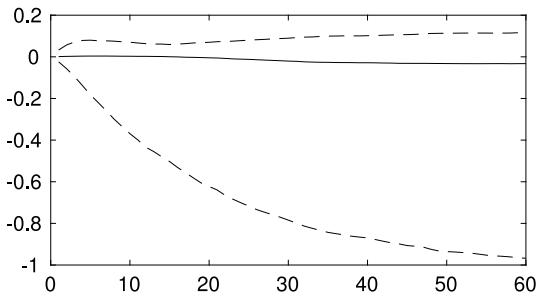

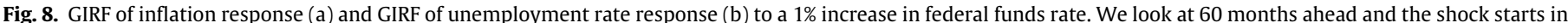
December 2007. Solid line is the 50th percentile and dashed line the $95 \%$ credible interval.

outperforms BVAR(1) at horizon 2, and 4 for all variables and horizon 1 for variable 1 .

Tables 5-8 display the log-predictive scores and the RMSEs for the growth rates of the US and UK data respectively. The BayesNP$\operatorname{VAR}(1)$ is on average the model with the better out-of-sample predictive performance for both data sets. The BayesNP-VAR(1) model outperforms the TVP-SV-VAR(1) for all forecasting horizons in both the overall and marginal log-predictive scores, and RMSEs with the US growth rates data. In terms of overall log-predictive scores it is slightly outperformed by the BVAR(3) at horizon 1, but it provides superior overall log-predictive scores for the longer time horizons of 2 and 4 months than any of the alternative models. The BayesNP-VAR outperforms the BVAR models for most variables at horizons 1, 2 and 4 in log predictive scores and for all variables, except unemployment rate, at all horizons with RMSE. In the case of the UK growth rates, the BayesNP-VAR(1) outperforms the other models for the overall and marginal log-predictive scores and RMSE for all horizons and all variables (with a few exceptions).

Tables 9-12 display the log-predictive scores and RMSEs for the levels of the US and UK data respectively. Once again, the BayesNPVAR(1) model produces better overall and marginal log-predictive scores and RMSEs for all time horizons when compared to the 
Table 3

Log-predictive scores and RMSEs for the simulated $\operatorname{VAR}(2)$ data.

\begin{tabular}{|c|c|c|c|c|c|c|c|c|}
\hline \multirow[t]{2}{*}{ Model } & \multirow[t]{2}{*}{ Horizon } & \multicolumn{4}{|c|}{ Joint and marginal scores } & \multicolumn{3}{|c|}{ RMSEs } \\
\hline & & Overall & Var1 & Var2 & Var3 & Var1 & Var2 & Var3 \\
\hline \multirow[t]{3}{*}{$\operatorname{BVAR}(1)$} & 1 & 123 & 49 & 42 & 47 & 0.52 & 0.58 & 0.62 \\
\hline & 2 & 135 & 44 & 48 & 50 & 0.57 & 0.65 & 0.69 \\
\hline & 4 & 138 & 50 & 52 & 51 & 0.70 & 0.73 & 0.72 \\
\hline \multirow[t]{3}{*}{$\operatorname{BVAR}(2)$} & 1 & 127 & 40 & 42 & 47 & 0.55 & 0.58 & 0.63 \\
\hline & 2 & 138 & 44 & 48 & 50 & 0.60 & 0.66 & 0.69 \\
\hline & 4 & 141 & 50 & 52 & 51 & 0.71 & 0.74 & 0.74 \\
\hline \multirow[t]{3}{*}{ BVAR(3) } & 1 & 129 & 41 & 43 & 50 & 0.55 & 0.59 & 0.67 \\
\hline & 2 & 142 & 50 & 50 & 52 & 0.63 & 0.70 & 0.73 \\
\hline & 4 & 145 & 51 & 53 & 52 & 0.73 & 0.78 & 0.76 \\
\hline \multirow[t]{3}{*}{$\operatorname{BVAR}(4)$} & 1 & 134 & 43 & 44 & 50 & 0.60 & 0.62 & 0.68 \\
\hline & 2 & 150 & 48 & 52 & 53 & 0.70 & 0.76 & 0.74 \\
\hline & 4 & 150 & 52 & 54 & 52 & 0.78 & 0.82 & 0.76 \\
\hline \multirow[t]{3}{*}{ BayesNP-VAR(1) } & 1 & 125 & 39 & 43 & 47 & 0.51 & 0.57 & 0.61 \\
\hline & 2 & 133 & 44 & 48 & 50 & 0.57 & 0.63 & 0.69 \\
\hline & 4 & 136 & 49 & 51 & 50 & 0.69 & 0.73 & 0.72 \\
\hline \multirow[t]{3}{*}{ TV-SV-VAR(1) } & 1 & 170 & 47 & 63 & 71 & 0.63 & 0.68 & 0.68 \\
\hline & 2 & 177 & 50 & 80 & 78 & 0.58 & 0.96 & 0.82 \\
\hline & 4 & 193 & 61 & 87 & 87 & 6.17 & 8.83 & 6.75 \\
\hline
\end{tabular}

Table 4

Log-predictive scores for simulated threshold VAR(2) data.

\begin{tabular}{|c|c|c|c|c|c|c|c|c|}
\hline \multirow[t]{2}{*}{ Model } & \multirow[t]{2}{*}{ Horizon } & \multicolumn{4}{|c|}{ Joint and marginal scores } & \multicolumn{3}{|c|}{ RMSEs } \\
\hline & & Overall & Var1 & Var2 & Var3 & Var1 & Var2 & Var3 \\
\hline \multirow[t]{3}{*}{$\operatorname{BVAR}(1)$} & 1 & 305 & 74 & 97 & 60 & 1.08 & 1.83 & 0.86 \\
\hline & 2 & 335 & 92 & 97 & 67 & 1.33 & 1.56 & 0.93 \\
\hline & 4 & 361 & 95 & 109 & 63 & 1.43 & 1.72 & 0.96 \\
\hline \multirow[t]{3}{*}{$\operatorname{BVAR}(2)$} & 1 & 276 & 75 & 82 & 47 & 1.42 & 1.73 & 0.85 \\
\hline & 2 & 338 & 94 & 78 & 50 & 1.71 & 1.46 & 0.93 \\
\hline & 4 & 341 & 96 & 85 & 51 & 1.46 & 1.55 & 0.97 \\
\hline \multirow[t]{3}{*}{$\operatorname{BVAR}(3)$} & 1 & 291 & 77 & 77 & 63 & 1.38 & 1.89 & 0.97 \\
\hline & 2 & 322 & 94 & 74 & 68 & 1.57 & 1.84 & 1.04 \\
\hline & 4 & 323 & 94 & 82 & 56 & 1.44 & 1.69 & 0.96 \\
\hline \multirow[t]{3}{*}{ BVAR(4) } & 1 & 297 & 78 & 81 & 65 & 1.42 & 2.08 & 1.07 \\
\hline & 2 & 313 & 97 & 83 & 68 & 1.59 & 2.13 & 1.19 \\
\hline & 4 & 332 & 96 & 82 & 59 & 1.46 & 1.99 & 1.11 \\
\hline \multirow[t]{3}{*}{ BayesNP-VAR(1) } & 1 & 171 & 67 & 55 & 59 & 1.04 & 0.75 & 0.79 \\
\hline & 2 & 204 & 81 & 59 & 70 & 1.51 & 0.86 & 0.83 \\
\hline & 4 & 211 & 89 & 61 & 72 & 1.91 & 0.93 & 0.96 \\
\hline \multirow[t]{3}{*}{ TV-SV-VAR(1) } & 1 & 195 & 69 & 64 & 67 & 1.01 & 0.75 & 0.79 \\
\hline & 2 & 229 & 88 & 71 & 79 & 1.52 & 0.91 & 0.97 \\
\hline & 4 & 244 & 102 & 76 & 86 & 2.39 & 1.23 & 1.54 \\
\hline
\end{tabular}

TVP-SV-VAR(1) for both the US and UK data. When compared to the BVAR models, it performs better on the UK data. In the US data, the BayesNP-VAR(1) model outperforms BVAR(1) overall for horizons 1 and 2 but not horizon 4. The BVAR(1) and BayesNP-VAR(1) model each produce better predictions for some variables but there is no clearly superior model for all variables for log predictive scores but BayesNP-VAR(1) outperforms BVAR(1) for all variables with RMSE.

To summarise the BayesNP-VAR(1) outperforms the TVP-SVVAR(1) for all predictive horizons, in both the simulated and empirical data sets. The non-stationary TVP-SV-VAR accounts for nonlinearity in the conditional mean, and heteroskedasticity in the conditional variance and therefore one would expect it to be better suited to capturing the nonlinear dynamic relationships between variables, leading to better predictive performance. However, for all data sets considered, it is often outperformed by BVAR models. This may be due to fact that the TVP-SV-VAR does not separate the non-linearity in the conditional mean and heteroskedasticity from non-stationarity, which can be a problem when $y_{t}$ is a stationary process. The transition density of the TVP-SV-VAR depends on the mean $\mu_{t}$ and the covariance $\Sigma_{t}$ which are modelled by nonstationary processes. Therefore, when this transition density is not appropriately chosen then the TVP-SV-VAR will not do a good job in approximating the real transition density.

\section{Discussion}

This paper introduces a new approach to modelling multivariate time series. Using Bayesian nonparametric methods, we have shown how we can express both marginal and transition densities as infinite mixtures, leading to a flexible stationary model that allows for non-linearity in the conditional mean, heteroskedasticity in the conditional variance, and non-Gaussianity. Our empirical results, for both US and UK data, as well as simulated data, indicate that the BayesNP-VAR model outperforms TVP-SV-VAR at all time horizons and shows substantial improvements over BVAR at most time horizons and data sets. The empirical results illustrate that it is useful to allow changes in mixture components to depend on the observed lagged values. However, our model assumes stationarity, 
Table 5

Log-predictive scores for growth rates of US data.

\begin{tabular}{lllllllll}
\hline Model & Horizon & \multicolumn{1}{l}{ Joint and marginal scores } & & & & & \\
\hline \multirow{3}{*}{ BVAR(1) } & & Overall & FEDFUNDS & INDPRO & LTIR & PAYEMS & PCEPI & UNRATE \\
& 1 & 314 & 13 & 159 & -10 & 87 & 96 & -23 \\
& 2 & 391 & 30 & 157 & 10 & 90 & 98 & -5 \\
BVAR(2) & 4 & 491 & 50 & 152 & 34 & 88 & 94 & 33 \\
& 1 & 306 & 9 & 160 & -10 & 82 & 95 & -25 \\
BVAR(3) & 2 & 392 & 35 & 158 & 14 & 82 & 99 & -11 \\
& 4 & 540 & 75 & 152 & 50 & 85 & 95 & 15 \\
BVAR(4) & 1 & 301 & 8 & 159 & -11 & 81 & 95 & -25 \\
& 2 & 387 & 36 & 158 & 15 & 81 & 99 & -11 \\
& 4 & 511 & 72 & 152 & 44 & 83 & 95 & 13 \\
BNP-VAR(1) & 1 & 301 & 8 & 159 & -10 & 81 & 95 & -25 \\
& 2 & 392 & 36 & 158 & 16 & 81 & 98 & -11 \\
& 4 & 511 & 72 & 152 & 45 & 83 & 95 & 13 \\
TV-SV-VAR(1) & 1 & 322 & 52 & 109 & 39 & 48 & 52 & 22 \\
& 2 & 335 & 51 & 107 & 38 & 47 & 51 & 21 \\
& 4 & 321 & 58 & 102 & 36 & 45 & 49 & 20
\end{tabular}

Table 6

RMSEs for growth rates of US data.

\begin{tabular}{llllllll}
\hline Model & Horizon & RMSES & & & & & \\
\hline \multirow{3}{*}{ BVAR(1) } & & FEDFUNDS & INDPRO & LTIR & PAYEMS & PCEPI & UNRATE \\
& 1 & 0.117 & 5.91 & 0.153 & 0.93 & 1.71 & 0.148 \\
& 2 & 0.189 & 5.92 & 0.238 & 0.83 & 1.88 & 0.199 \\
BVAR(2) & 4 & 0.307 & 6.04 & 0.326 & 0.87 & 1.77 & 0.251 \\
& 1 & 0.130 & 6.33 & 0.161 & 0.73 & 1.72 & 0.145 \\
BVAR(3) & 2 & 0.233 & 6.46 & 0.254 & 0.66 & 1.96 & 0.184 \\
& 4 & 0.390 & 6.58 & 0.359 & 0.70 & 1.86 & 0.214 \\
BVAR(4) & 1 & 0.125 & 6.23 & 0.163 & 0.75 & 1.69 & 0.144 \\
& 2 & 0.209 & 6.41 & 0.249 & 0.70 & 1.92 & 0.182 \\
BayesNP-VAR(1) & 4 & 0.343 & 6.66 & 0.323 & 0.69 & 1.84 & 0.204 \\
& 1 & 0.127 & 6.17 & 0.166 & 0.76 & 1.69 & 0.144 \\
& 2 & 0.217 & 6.39 & 0.255 & 0.74 & 1.92 & 0.185 \\
TV-SV-VAR(1) & 2 & 0.385 & 6.65 & 0.336 & 0.72 & 1.91 & 0.209 \\
& 4 & 0.026 & 4.94 & 0.153 & 0.69 & 1.67 & 0.159 \\
& 1 & 0.043 & 5.05 & 0.232 & 0.67 & 1.81 & 0.228 \\
& 2 & 0.061 & 5.10 & 0.328 & 0.74 & 1.76 & 0.332
\end{tabular}

which is a strong assumption about macroeconomic data. There may be benefits in terms of predictive power from relaxing this assumption by allowing both marginal and transition densities to vary over time. We will investigate these types of models in future work.

\section{Appendix A. Gibbs sampler}

We assume that data $y_{1}, \ldots, y_{T}$ are observed and we fit the model with $L$ lags using the following Gibbs sampler. We use the renormalised stick-breaking construction of Griffin (2016) with $K$ atoms as our truncation. This implies a transition density of the form

$p\left(y_{t} \mid y_{(t-L):(t-1)}\right)=\frac{\sum_{j=1}^{\infty} w_{j} k\left(y_{(t-L): t} \mid \theta_{j}\right)}{\sum_{j=1}^{\infty} w_{j} k\left(y_{(t-L):(t-1)} \mid \theta_{j}\right)}$

where $w_{j}=V_{j} \prod_{m<j}\left(1-V_{m}\right)$ and $V_{j} \stackrel{i i d}{\sim} \mathrm{Be}(1, M)$ for $1 \leq k \leq K$ and $\theta_{j} \stackrel{i i d}{\sim} H$.
Updating $\mu$

The full conditional density of $\mu_{j, i}$ is proportional to

$\exp \left\{-\frac{\left(\mu_{j, i}-\mu_{0, i}\right)^{2}}{2 \sigma_{0, i}^{2}}\right\} \prod_{t=L+1}^{T} p_{K}\left(y_{t} \mid y_{(t-L):(t-1)}\right)$.

The parameter can be updated using an adaptive random walk Metropolis-Hastings sampler where a normal proposal is used whose variance is tuned to have an acceptance rate 0.234 .

Updating $S$

The full conditional density of $S_{j, i}$ is proportional to

$s_{j, i}^{-\left(1+\alpha_{S}\right)} \exp \left\{-\zeta_{i}\left(\alpha_{S}-1\right) / s_{j, i}\right\} \prod_{t=L+1}^{T} p_{K}\left(y_{t} \mid y_{(t-L):(t-1)}\right)$.

The parameter can be updated using an adaptive random walk Metropolis-Hastings sampler on the log scale where a normal 
Table 7

Log-predictive scores for growth rates of UK data.

\begin{tabular}{llllrrrr}
\hline Model & Horizon & \multicolumn{2}{l}{ Joint and marginal scores } & & & \\
\hline \multirow{3}{*}{ BVAR(1) } & & Overall & CPI & INDPRO & LTIR & STIR & UNRATE \\
& 1 & 254 & 121 & 183 & -1 & 7 & -48 \\
& 2 & 338 & 120 & 181 & 20 & 23 & -25 \\
BVAR(2) & 4 & 485 & 115 & 173 & 55 & 41 & 23 \\
& 1 & 235 & 116 & 182 & -5 & 4 & -55 \\
BVAR(3) & 2 & 356 & 114 & 180 & 23 & 29 & -20 \\
& 4 & 647 & 111 & 174 & 72 & 65 & 72 \\
BVAR(4) & 1 & 231 & 115 & 182 & -5 & 4 & -57 \\
& 2 & 346 & 112 & 180 & 24 & 30 & -32 \\
& 4 & 608 & 111 & 173 & 66 & 64 & 60 \\
BayesNP-VAR(1) & 1 & 224 & 114 & 182 & -4 & 3 & -61 \\
& 2 & 327 & 111 & 180 & 24 & 29 & -40 \\
& 4 & 572 & 109 & 173 & 67 & 60 & 27 \\
TV-SV-VAR(1) & 2 & 168 & 104 & 145 & -12 & -42 & -67 \\
& 4 & 220 & 95 & 144 & 12 & -22 & -42 \\
& 1 & 345 & 93 & 140 & 3 & -27 & -12
\end{tabular}

Table 8

RMSEs for growth rates of UK data.

\begin{tabular}{|c|c|c|c|c|c|c|}
\hline \multirow[t]{2}{*}{ Model } & \multirow[t]{2}{*}{ Horizon } & \multicolumn{5}{|c|}{ RMSEs } \\
\hline & & CPI & INDPRO & LTIR & STIR & UNRATE \\
\hline \multirow[t]{3}{*}{$\operatorname{BVAR}(1)$} & 1 & 3.24 & 10.19 & 0.20 & 0.058 & 0.076 \\
\hline & 2 & 3.58 & 10.52 & 0.35 & 0.108 & 0.130 \\
\hline & 4 & 3.51 & 10.32 & 0.58 & 0.205 & 0.237 \\
\hline \multirow[t]{3}{*}{ BVAR(2) } & 1 & 2.87 & 9.94 & 0.18 & 0.123 & 0.069 \\
\hline & 2 & 2.89 & 10.22 & 0.32 & 0.224 & 0.115 \\
\hline & 4 & 3.06 & 10.46 & 0.56 & 0.364 & 0.199 \\
\hline \multirow[t]{3}{*}{ BVAR(3) } & 1 & 2.75 & 9.94 & 0.18 & 0.126 & 0.070 \\
\hline & 2 & 2.75 & 10.22 & 0.33 & 0.248 & 0.104 \\
\hline & 4 & 3.00 & 10.42 & 0.56 & 0.417 & 0.169 \\
\hline \multirow[t]{3}{*}{ BVAR(4) } & 1 & 2.64 & 9.96 & 0.20 & 0.131 & 0.062 \\
\hline & 2 & 2.54 & 10.21 & 0.37 & 0.253 & 0.092 \\
\hline & 4 & 2.70 & 10.50 & 0.65 & 0.509 & 0.159 \\
\hline \multirow[t]{3}{*}{ BayesNP-VAR(1) } & 1 & 2.29 & 9.93 & 0.18 & 0.064 & 0.089 \\
\hline & 2 & 2.12 & 9.94 & 0.30 & 0.112 & 0.158 \\
\hline & 4 & 1.99 & 10.04 & 0.49 & 0.198 & 0.295 \\
\hline \multirow[t]{3}{*}{ TV-SV-VAR(1) } & 1 & 2.57 & 14.33 & 0.19 & 0.084 & 0.087 \\
\hline & 2 & 2.07 & 15.23 & 0.32 & 0.148 & 0.126 \\
\hline & 4 & 2.11 & 13.59 & 0.59 & 0.542 & 0.380 \\
\hline
\end{tabular}

proposal is used whose variance is tuned to have an acceptance rate 0.234 .

\section{Updating $\xi$}

The full conditional density of $\xi_{j, i}$ is proportional to

$\xi_{j, i}^{v / 2-1} \exp \left\{-\frac{v}{2} \xi_{j, i}\right\} \prod_{t=L+1}^{T} p_{K}\left(y_{t} \mid y_{(t-L):(t-1)}\right)$.

The parameter can be updated using an adaptive random walk Metropolis-Hastings sampler on the log scale where a normal proposal is used whose variance is tuned to have an acceptance rate 0.234 .

\section{Updating $\rho$}

The full conditional density of $\rho_{j, i}$ is proportional to

$$
\prod_{t=L+1}^{T} p_{K}\left(y_{t} \mid y_{(t-L):(t-1)}\right) \text {. }
$$

The parameter can be updated using an adaptive random walk Metropolis-Hastings sampler on a logit scale where a normal proposal is used whose variance is tuned to have an acceptance rate 0.234 .

Updating $\rho^{\star}$

The full conditional density of $\rho_{j, i}^{\star}$ is proportional to $\prod_{t=L+1}^{T} p_{K}\left(y_{t} \mid y_{(t-L):(t-1)}\right)$.

The parameter can be updated using an adaptive random walk Metropolis-Hastings sampler on a logit scale where a normal proposal is used whose variance is tuned to have an acceptance rate 0.234 .

Updating $\Lambda$

The full conditional density of $\Lambda_{j, i, k}$ is proportional to

$\exp \left\{-\frac{1}{2} \Lambda_{j, i, k}^{2} \tau_{j, k} \phi_{j, i, k}\right\} \prod_{t=L+1}^{T} p_{K}\left(y_{t} \mid y_{(t-L):(t-1)}\right)$. 
Table 9

Log-predictive scores for levels of US data.

\begin{tabular}{|c|c|c|c|c|c|c|c|c|}
\hline \multirow[t]{2}{*}{ Model } & \multirow[t]{2}{*}{ Horizon } & \multicolumn{7}{|c|}{ Joint and marginal scores } \\
\hline & & Overall & FEDFUNDS & INDPRO & LTIR & PAYEMS & PCEPI & UNRATE \\
\hline \multirow[t]{3}{*}{$\operatorname{BVAR}(1)$} & 1 & 327 & 14 & 159 & -9 & 98 & 98 & -20 \\
\hline & 2 & 489 & 30 & 173 & 8 & 118 & 119 & -2 \\
\hline & 4 & 793 & 46 & 190 & 28 & 160 & 147 & 20 \\
\hline \multirow[t]{3}{*}{$\operatorname{BVAR}(2)$} & 1 & 309 & 9 & 157 & -10 & 88 & 94 & -25 \\
\hline & 2 & 533 & 35 & 177 & 14 & 116 & 130 & -7 \\
\hline & 4 & 1057 & 75 & 216 & 47 & 186 & 199 & 26 \\
\hline \multirow[t]{3}{*}{$\operatorname{BVAR}(3)$} & 1 & 301 & 8 & 158 & -11 & 82 & 94 & -26 \\
\hline & 2 & 504 & 36 & 178 & 14 & 104 & 130 & -12 \\
\hline & 4 & 967 & 69 & 216 & 42 & 170 & 201 & 16 \\
\hline \multirow[t]{3}{*}{$\operatorname{BVAR}(4)$} & 1 & 298 & 8 & 157 & -10 & 82 & 94 & -27 \\
\hline & 2 & 505 & 36 & 177 & 16 & 102 & 132 & -13 \\
\hline & 4 & 944 & 71 & 213 & 44 & 161 & 200 & 13 \\
\hline \multirow[t]{3}{*}{ BayesNP-VAR(1) } & 1 & 290 & -9 & 210 & 22 & 216 & 178 & 3 \\
\hline & 2 & 486 & 15 & 231 & 39 & 257 & 204 & 19 \\
\hline & 4 & 899 & 43 & 264 & 47 & 291 & 246 & 27 \\
\hline \multirow[t]{3}{*}{ TV-SV-VAR(1) } & 1 & 930 & 41 & 280 & 36 & 262 & 298 & 14 \\
\hline & 2 & 963 & 51 & 285 & 42 & 266 & 303 & 19 \\
\hline & 4 & 996 & 58 & 292 & 47 & 275 & 312 & 25 \\
\hline
\end{tabular}

Table 10

RMSEs for levels of US data.

\begin{tabular}{llllllll}
\hline Model & Horizon & RMSES & & & & \\
\hline & & FEDFUNDS & INDPRO & LTIR & PAYEMS & PCEPI & UNRATE \\
BVAR(1) & 1 & 0.112 & 4.91 & 0.149 & 1.73 & 1.78 & 0.160 \\
& 2 & 0.215 & 7.12 & 0.223 & 3.38 & 2.84 & 0.229 \\
& 4 & 0.400 & 11.01 & 0.306 & 6.67 & 3.76 & 0.330 \\
BVAR(2) & 1 & 0.126 & 5.59 & 0.161 & 1.23 & 1.67 & 0.143 \\
& 2 & 0.222 & 8.41 & 0.251 & 2.39 & 2.94 & 0.194 \\
BVAR(3) & 4 & 0.336 & 13.10 & 0.335 & 5.00 & 4.16 & 0.259 \\
& 1 & 0.125 & 5.73 & 0.159 & 0.91 & 1.68 & 0.138 \\
BVAR(4) & 2 & 0.212 & 8.97 & 0.239 & 1.47 & 2.97 & 0.177 \\
& 4 & 0.273 & 14.82 & 0.305 & 2.94 & 4.17 & 0.203 \\
BayesNP-VAR(1) & 1 & 0.128 & 5.52 & 0.168 & 0.86 & 1.68 & 0.136 \\
& 2 & 0.211 & 8.53 & 0.255 & 1.33 & 3.01 & 0.174 \\
& 4 & 0.318 & 13.90 & 0.309 & 2.49 & 4.29 & 0.199 \\
TV-SV-VAR(1) & 1 & 0.031 & 4.91 & 0.149 & 1.17 & 2.03 & 0.158 \\
& 4 & 0.057 & 7.14 & 0.224 & 2.23 & 3.49 & 0.224 \\
& 2 & 0.093 & 10.28 & 0.304 & 4.40 & 5.82 & 0.324
\end{tabular}

The parameter can be updated using an adaptive random walk Metropolis-Hastings sampler where a normal proposal is used whose variance is tuned to have an acceptance rate 0.234 .

\section{Updating $\phi$}

The full conditional distribution for $\phi_{j, i, k}$ is $\mathrm{Ga}((\nu+1) / 2$, $\left.\left(v+\tau_{j, k} \Lambda_{j, i, k}\right) / 2\right)$.

\section{Updating $\delta$}

Let $\tau_{j, k}^{(h)}=\prod_{t=1, t \neq h}^{k} \delta_{t}$. The full conditional distribution for $\delta_{j, 1}$ is $\mathrm{Ga}\left(a_{1}+p q / 2,1+\sum_{k=1}^{q} \tau_{j, k}^{(1)} \sum_{i=1}^{p} \phi_{j, i, k} \lambda_{j, i, k}^{2}\right)$.

The full conditional distribution for $\delta_{h}, 2 \leq k \leq q$ is

$\mathrm{Ga}\left(a_{2}+p(q-h+1) / 2,1+\sum_{k=h}^{q} \tau_{j, k}^{(h)} \sum_{i=1}^{p} \phi_{j, i, k} \lambda_{j, i, k}^{2}\right)$.
Updating $\zeta$

The full conditional distribution of $\zeta_{i}$ is Ga $\left(K \alpha_{S}+\kappa_{1},\left(\alpha_{S}-1\right)\right.$ $\left.\sum_{j=1}^{K} s_{j, i}+\kappa_{2}\right)$.

Updating $V$

The full conditional density of $V_{j}$ is proportional to

$\left(1-V_{j}\right)^{M-1} \prod_{t=L+1}^{T} p_{K}\left(y_{t} \mid y_{(t-L):(t-1)}\right)$.

The parameter can be updated using an adaptive random walk Metropolis-Hastings sampler on the logit scale where a normal proposal is used whose variance is tuned to have an acceptance rate 0.234 .

Updating $M$

The full conditional distribution of $M$ is Ga $\left(1+K, 1-\sum_{i=1}^{K}\right.$ $\left.\log \left(1-V_{i}\right)\right)$. 
Table 11

Log-predictive scores for levels of UK data.

\begin{tabular}{|c|c|c|c|c|c|c|c|}
\hline \multirow[t]{2}{*}{ Model } & \multirow[t]{2}{*}{ Horizon } & \multicolumn{6}{|c|}{ Joint and marginal scores } \\
\hline & & Overall & CPI & INDPRO & LTIR & STIR & UNRATE \\
\hline \multirow[t]{3}{*}{$\operatorname{BVAR}(1)$} & 1 & 313 & 128 & 184 & -3 & 10 & -8 \\
\hline & 2 & 620 & 171 & 197 & 16 & 27 & 116 \\
\hline & 4 & 1498 & 305 & 207 & 41 & 49 & 481 \\
\hline \multirow[t]{3}{*}{$\operatorname{BVAR}(2)$} & 1 & 253 & 121 & 183 & -6 & 3 & -45 \\
\hline & 2 & 503 & 154 & 189 & 20 & 28 & 16 \\
\hline & 4 & 1261 & 250 & 194 & 54 & 61 & 239 \\
\hline \multirow[t]{3}{*}{$\operatorname{BVAR}(3)$} & 1 & 236 & 118 & 181 & -6 & 4 & -53 \\
\hline & 2 & 430 & 145 & 188 & 21 & 29 & -23 \\
\hline & 4 & 972 & 233 & 191 & 57 & 58 & 90 \\
\hline \multirow[t]{3}{*}{$\operatorname{BVAR}(4)$} & 1 & 226 & 116 & 181 & -6 & 4 & -60 \\
\hline & 2 & 399 & 141 & 188 & 20 & 28 & -38 \\
\hline & 4 & 853 & 230 & 190 & 54 & 56 & 42 \\
\hline \multirow[t]{3}{*}{ BayesNP-VAR(1) } & 1 & 193 & 124 & 177 & -13 & -55 & -46 \\
\hline & 2 & 329 & 155 & 199 & 19 & -12 & -19 \\
\hline & 4 & 561 & 190 & 210 & 59 & 19 & 34 \\
\hline \multirow[t]{3}{*}{ TV-SV-VAR(1) } & 1 & 617 & 270 & 222 & 47 & 50 & 28 \\
\hline & 2 & 641 & 276 & 224 & 52 & 57 & 33 \\
\hline & 4 & 673 & 284 & 229 & 59 & 66 & 40 \\
\hline
\end{tabular}

Table 12

RMSEs for levels of UK data.

\begin{tabular}{lllllll}
\hline Model & Horizon & RMSEs & & & & \\
\hline \multirow{3}{*}{ BVAR(1) } & & CPI & INDPRO & LTIR & STIR & UNRATE \\
& 1 & 4.09 & 10.01 & 0.173 & 0.281 & 0.213 \\
& 2 & 7.78 & 13.81 & 0.288 & 0.541 & 0.408 \\
BVAR(2) & 4 & 15.42 & 16.62 & 0.459 & 1.005 & 0.770 \\
& 1 & & & & & 0.135 \\
& 2 & 3.36 & 10.20 & 0.164 & 0.114 & 0.296 \\
BVAR(3) & 4 & 6.26 & 12.69 & 0.270 & 0.272 & 0.643 \\
& 1 & 12.73 & 15.11 & 0.411 & 0.700 & 0.094 \\
BVAR(4) & 2 & 3.13 & 9.71 & 0.166 & 0.124 & 0.170 \\
& 4 & 5.70 & 12.31 & 0.280 & 0.259 & 0.381 \\
& 1 & 11.74 & 14.87 & 0.444 & 0.497 & 0.072 \\
BayesNP-VAR(1) & 2 & 2.99 & 9.69 & 0.164 & 0.120 & 0.118 \\
& 4 & 5.43 & 12.22 & 0.271 & 0.239 & 0.240 \\
& 1 & 11.41 & 14.12 & 0.425 & 0.453 & 0.085 \\
TV-SV-VAR(1) & 2 & 2.81 & 9.30 & 0.172 & 0.039 & 0.149 \\
& 4 & 4.89 & 11.96 & 0.285 & 0.074 & 0.271
\end{tabular}

\section{Appendix B. Simulated VAR(2) specification}

$$
\begin{aligned}
y_{t}= & \left(\begin{array}{l}
0.13 \\
0.12 \\
0.29
\end{array}\right)+\left(\begin{array}{ccc}
0.39 & 0.10 & 0.05 \\
0.35 & 0.34 & 0.47 \\
0.49 & 0.24 & 0.24
\end{array}\right) y_{t-1} \\
& +\left(\begin{array}{ccc}
0.06 & 0.11 & 0.02 \\
-0.19 & -0.18 & -0.01 \\
-0.31 & -0.13 & 0.09
\end{array}\right) y_{t-2}+e_{t}
\end{aligned}
$$

with covariance matrix,

$$
\Sigma=\left(\begin{array}{lll}
0.28 & 0.03 & 0.07 \\
0.03 & 0.29 & 0.14 \\
0.07 & 0.14 & 0.36
\end{array}\right) \text {. }
$$

\section{References}

Andrieu, C., Moulines, E., 2006. On the ergodicity properties of some adaptive MCMC algorithms. Ann. Appl. Probab. 16, 1462-1505.
Antoniano-Villalobos, I., Walker, S.G., 2016. A nonparametric model for stationary time series. J. Time Series Anal. 37, 126-142.

Atchadé, Y.F., Fort, G., 2010. Limit theorems for some adaptive MCMC algorithms with subgeometric kernels. Bernoulli 16, 116-154.

Atchadé, Y.F., Rosenthal, J.S., 2005. On adaptive Markov chain Monte Carlo algorithms. Bernoulli 11, 815-828.

Auerbach, A.J., Gorodnichenko, Y., 2013. Fiscal multipliers in recession and expansion. In: Alesina, A., Giavazzi, F. (Eds.), Fiscal Policy after the Financial Crisis, no. 63-98. University of Chicago Press.

Baum, A., Koester, G.B., 2011. The Impact of Fiscal Policy on Economic Activity Over the Business Cycle: Evidence from a thershold VAR analysis, Discussion paper, Series 1 Economic Studies N03/2011. Deutsche Bundesbank.

Beaudry, P., Koop, G., 1993. Do recessions permantly change output?. J. Monet. Econ. 31, 149-163.

Bhattacharya, A., Dunson, D.B., 2011. Sparse Bayesian infinite factor models. Biometrika 98 (2), 291-306.

Carriero, A., Clarke, T.E., Marcellino, M., 2015. Bayesian VARs: Specification choices and forecasting accuracy. J. Appl. Econometrics 30, 46-73.

Chauvet, M., 1998. An econometric characterization of business cycle dynamics with factor structure and regime switiching. Internat. Econom. Rev. 39, 969-996. 
Chib, S., 1996. Calculating posterior distributions and model estimates in Markov mixture models. J. Econometrics 75, 79-97.

Cogley, T., Sargent, T.J., 2001. Evolving post world war II US inflation dynamics. In: NBER Macroeconomics Annual, vol. 16. pp. 331-338.

Cogley, T., Sargent, T.J., 2005a. Drifts and volatilities: Monetary policies and outcomes in the post WWII US. Rev. Econ. Dyn. 8, 262-302.

Dahl, C.M., Gonzalez-Rivera, G., 2003a. Identifying nonlinear components by random fields in the US GNP growth. Implications and shape of the business Cycle. Stud. Nonlinear Dyn. Econ. 7.

Dahl, C.M., Gonzalez-Rivera, G., 2003b. Testing of negleted nonlinearity in regression models based on the theory of random fields. J. Econometrics 114, $141-164$.

Diebold, F.X., Rudebusch, G.D., 2001. Five questions about business cycles. FRBSF Econ. Rev. 1-15.

Gambacorta, L., Hofmann, B., Peersman, G., 2014. The effectiveness of unconventional monetary policy at the zero lower bound: A cross-country analysis. J. Money Credit Bank. 46, 615-642.

Geweke, J., Keane, M., 2007. Smoothly mixing regressions. J. Econometrics 138, 252-290.

Granger, C., Terasvirta, T., 1994. Modelling nonlinear economic relationships. Int. J. Forecast. 10, 169-171.

Griffin, J.E., 2016. An adaptive truncation method for inference in Bayesian nonparametric models. Stat. Comput. 26, 423-441.

Hamilton, J.D., 1989. A new approach to the economic analysis of nonstationary time series and the business cycle. Econometrica 57, 357-384.

Hamilton, J.D., 2001. A parametric approach to flexible nonlinear inference. Econometrica 69, 537-573.

Hansen, B.E., 1992. The likelihood ratio test under nonstandard conditions: Testing the Markov switching model of GNP. J. Appl. Econometrics 7, 61-82.

Härdle, W., Tsybakov, A., Yang, L., 1998. Nonparametric vector autorgression. J. Statist. Plann. Inference 68, 221-245.

Hjort, N.L., Holmes, C., Müller, P., Walker, S.G. (Eds.), 2010. Bayesian Nonparametrics, first ed. In: Statistic and Probabilistic Mathematics, Cambridge University Press.

Hubrich, K., Teräsvirta, T., 2013. Thresholds and smooth transitions in vector autoregressive models. In: Fomby, T.B., Kilian, L., Murphy, A. (Eds.), VAR Models in Macroeconomics-New Developments and Applications: Essays in Honor of Christopher A. Sims. In: Advances in Econometrics, vol. 32, Emerald Group Publishing Limited.

Hyvärinen, A., Zhang, K., Shimizu, S., Hoyer, P.O., 2010. Estimation of a structural vector autoregression model using non-Gaussianity. J. Mach. Learn. Res. 11, 1709-1731.

Jacobs, R., Jordan, M., Nowlan, S., Hinton, G., 1991. Adaptive mixtures of local experts. Neural Comput. 3, 79-87.

Jeliazkov, I., 2013. Nonparametric vector autoregressions: Specification, estimation and inference. In: Fomby, T.B., Kilian, L., Murphy, A. (Eds.), VAR Models in Macroeconomics-New Developments and Applications: Essays in Honor of Christopher A. Sims. In: Advances in Econometrics, vol. 32, Emerald Group Publishing Limited.

Jordan, M., Jacobs, R., 1994. Hierarchical mixtures of experts and the EM algorithm. Neural Comput. 6, 181-214.

Kim, C.J., Morley, J., Piger, J., 2005. Nonlinearity and the permanent effects of recessions. J. Appl. Econometrics 20, 291-309.
Kim, C.J., Nelson, C.R., 1999. Has the us economy become more stable? a Bayesian approach based on the business cycle. Rev. Econ. Stat. 81, 608-616.

Koop, G., Korobilis, D., 2010. Bayesian multivariate time series methods for empirical macroeconomics. Found. Trends Econ. 3, 267-358.

Koop, G., Pesaran, M.H., Potter, S.M., 1996. Impluse response analysis in nonlinear multivariate models. J. Econometrics 74, 119-147.

Lanne, M., Lütkepohl, H., 2010. Structural vector autoregressions with nonnormal residuals. J. Bus. Econom. Statist. 28, 159-168.

Lanne, M., Meitz, M., Saikkonen, P., 2017. Identification and estimation of nonGaussian structural vector autoregressions. J. Econometrics 196, 288-304.

Lucas, R.E., 1980. Methods and problems in business cycle theory. J. Money Credit Bank. 12, 696-715

Matthes, C., Barnichon, R., 2015. Measuring The Nonlinear Effects of Monetary Policy, Number 49 in 2015 Meeting Papers. Society of Economic Dynamics.

Moneta, A., Entner, D., Hoyer, P.O., Coad, A., 2013. Causal inference by independent component analysis: theory and applications. Oxf. Bull. Econ. Stat. 75, 705-730.

Norets, A., Pati, D., 2017. Adaptive Bayesian estimation of conditional densities. Econometric Theory 33, 980-1012.

Pagan, A., 1997. Towards an understanding of some business cycle characteristics. Aust. Econ. Rev. 30, 1-15.

Pesaran, M.H., Potter, S.M., 1997. A floor and ceiling model of US output. J. Econom. Dynam. Control 21, 661-695.

Potter, S.M., 1995. A non linear approach to US GNP. J. Appl. Econometrics 10 $109-125$.

Primiceri, G., 2005. Time varying structural vector autoregressions and monetary policy. Rev. Econom. Stud. 72, 821-852.

Ravn, M.O., Sola, M., 2004. Asymmetric effects of monetary policy in the US. Fed. Reserve Bank St. Louis Rev. 86, 41-60.

Sethuraman, J., 1994. A constructive definition of Dirichlet priors. Statist. Sinica 4 (2), 639-650.

Sims, C., 1980. Macroeconomics and reality. Econometrica 48 (1), 1-48.

Sims, C., Zha, T., 2006. Were there regime switches in us monetary policy?. Am. Econ. Rev. 96, 54-81.

Sörensen, B.E., Wu, L., Yosha, O., 2001. Output fluctuations and fiscal policy. Eur. Econ. Rev. 45, 1271-1310.

Stock, J., Watson, M., 1996. Evidence of structural instability in macroeconomic time series relations. J. Bus. Econom. Statist. 14, 11-30.

Stock, J., Watson, M., 1999. Handbook of Macroeconomics, vol. 1, Part A. Elsevier, Chapter 1: Business cycle fluctuations in US macroeconomic time series.

Stock, J., Watson, M., 2001. Vector autoregressions. J. Econ. Perspect. 15, 101-116.

Stock, J., Watson, M., 2002. Macroeconomic forecasting using diffusion indexes. J. Bus. Econom. Statist. 20, 147-162.

Teräsvirta, T., 1994. Specification, estimation and evaluation of smooth transition autoregressive models. J. Amer. Statist. Assoc. 89, 208-218.

Villani, M., Kohn, R., Giordani, P., 2009. Regression density estimation using smooth adaptive Gaussian mixtures. J. Econometrics 153, 155-173.

Villani, M., Kohn, R., Nott, D., 2012. Generalized smooth finite mixtures. J. Econometrics 171, 121-133.

Weise, C.L., 1999. The asymmetric effects of monetary policy: A non linear vector autoregression approach. J. Money Credit Bank. 31, 85-108. 